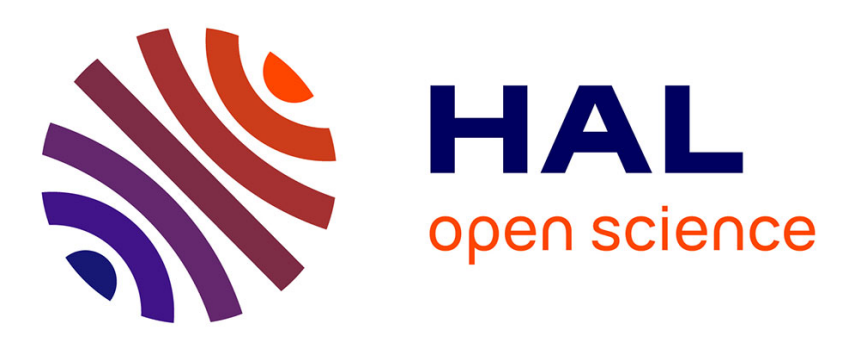

\title{
Dynamics of rearrangements during inclination of granular packings: the avalanche precursor regime
}

Sebastien Kiesgen de Richter, Renaud Delannay, Gérard Le Caër

\section{To cite this version:}

Sebastien Kiesgen de Richter, Renaud Delannay, Gérard Le Caër. Dynamics of rearrangements during inclination of granular packings: the avalanche precursor regime. Journal of Statistical Mechanics: Theory and Experiment, 2012, 2012 (4), pp.04013. 10.1088/1742-5468/2012/04/P04013 . hal00909253

\section{HAL Id: hal-00909253 https://hal.science/hal-00909253}

Submitted on 19 Oct 2017

HAL is a multi-disciplinary open access archive for the deposit and dissemination of scientific research documents, whether they are published or not. The documents may come from teaching and research institutions in France or abroad, or from public or private research centers.
L'archive ouverte pluridisciplinaire HAL, est destinée au dépôt et à la diffusion de documents scientifiques de niveau recherche, publiés ou non, émanant des établissements d'enseignement et de recherche français ou étrangers, des laboratoires publics ou privés. 


\title{
Dynamics of rearrangements during inclination of granular packings: the avalanche precursor regime
}

\author{
S Kiesgen de Richter ${ }^{1}$, G Le Caër ${ }^{2}$ and R Delannay ${ }^{2}$ \\ ${ }^{1}$ Université de Lorraine and CNRS, LEMTA, UMR 7563, \\ Vandoeuvre-les-Nancy, F-54500, France \\ ${ }^{2}$ Institut de Physique de Rennes-UMR UR1-CNRS 6251, Université de \\ Rennes 1, Bâtiment 11A, Campus de Beaulieu, 263 avenue Général Leclerc, \\ 35042, Rennes Cedex, France \\ E-mail: sebastien.kiesgen@ensem.inpl-nancy.fr, gerard.le-caer@univ-rennes1.fr \\ and renaud.delannay@univ-rennes1.fr
}

\begin{abstract}
The dynamics of inclined 3D granular packings driven towards their stability limits is studied experimentally through the dynamics of rearrangements of grains at their surfaces. Two regimes are identified in that way: a regime of limited and independent rearrangements of grains at small angles and a regime of extended events, called 'precursors of avalanches', at large angles. Both regimes are characterized. The transition between the two regimes is attributed to the emergence of slidings smaller than one grain diameter at the surface of the packing with a stickslip dynamics which reflects the emergence of long-range correlations at the approach of the avalanche. The observed superficial events are shown to depend strongly on the microscopic details of the interaction between grains. In addition to the study of precursors, we discuss the consequences of this macroscopic correlation length on the packing stability. We show in particular that the avalanche angle $\theta_{\mathrm{a}}$ depends not only on the width of the packing but also on its length.
\end{abstract}

Keywords: granular matter, avalanches (experiment), fracture (experiment), sandpile models (experiment)

\section{Introduction}

The existence of an unjamming transition is an essential characteristic of granular matter [21,33, 16, 24, 9,43]. Liu and Nagel [22] proposed a conceptual phase diagram for jammed systems which holds for glassy, pasty, or granular materials whose mechanical behaviours are indeed similar. These materials are jammed at rest and can sustain normal loads and shear stresses, but if a threshold shear stress is exceeded, part of the material starts to flow with properties that appear to differ from those of a Newtonian fluid. The discrete nature of granular materials renders their behaviour very complex. In response to some change in external forces, the macroscopic activity of a granular system is related 
to the evolving structure of the pile, and more specifically the evolving geometry of its contact network, and to the nature of the contacts themselves (frictional interactions, sliding or not). Such complex behaviours are of great interest for both industrial and natural processes.

The mechanical rigidity of granular packings is related to the field of rheology close to dynamical arrest. Some examples are drilling fluids for the oil industry but also cement, concrete and powders of all kinds. Granular media, foams, and emulsions are archetypes of jammed systems whose rheological properties have been thoroughly investigated (see for example $[27,11,23,7])$. These systems are thought to exhibit a yielding behaviour, they do not flow under small applied shear stresses and reach a velocity which continuously increases with the difference between the applied stress and the yield stress [39,12]. In Nature, geological processes like landslides and rock avalanches also involve an unjamming transition of granular media. Moreover, many natural phenomena, like earthquakes, involve interacting elements which discharge when they reach a trigger threshold, and become quiescent again. The activity of a discharging element stimulates neighbouring elements. The corresponding models are closely related to avalanche models on a formal level $[4,34]$. For free surface flows of granular systems under the action of gravity, the 'jamming transition' above a critical shear stress is simply evidenced by the existence of the angle of maximum stability of a pile, $\theta_{\mathrm{a}}$, associated with internal friction [46]. After the flow starts, the angle of the pile relaxes towards the angle of repose, $\theta_{\mathrm{r}}$, smaller than $\theta_{\mathrm{a}}$. A hysteretic behaviour naturally results from the fact that $\theta_{\mathrm{r}}<\theta_{\mathrm{a}}$. Consequently, two limiting curves exist for the solid-liquid transition, depending on whether the flow starts or stops [36]. Similar observations were made for granular flows in a rotating drum as a function of the rotation rate [13]. These studies were performed in stationary conditions where the system was submitted to a series of statistically similar jamming and unjamming transitions. By contrast, the behaviour of inclined 3D granular media was investigated experimentally in non-stationary conditions, showing that parameters like humidity, system dimensions, friction between grains, bottom roughness, or packing fraction can influence the value of the maximum angle of stability of a packing $[2,3,5]$. Aguirre et al [2] concluded that the influence of the number of grain layers on the stability of a packing is noticeable up to about ten layers, while it becomes independent of it for larger numbers of layers. The angle at which an avalanche starts was further found to increase with the packing fraction [3].

Exploration of the dynamical response of an inclined granular packing before the avalanche starts is of great interest as it allows a study of the dynamical transition from a static packing to a flowing one and brings information which may be helpful for prediction of the occurrence of an avalanche. Early experiments $[18,17,38]$ measured the flow past the rim of a sandpile; the technique was insensitive to sand slides that were entirely contained in the pile. Bretz et al [8] used a digital imaging technique to analyse the avalanches occurring during the slow inclination of a box. These large slides were separated by a sequence of rearrangements of the surface grains which were recorded by a camera. In its initial state, the sand pile was prepared by rotating the box until the rough substrate, covered with glued beads, began to appear at the top of the tray after some avalanches. By contrast, in [30]-[32] freshly prepared piles filling a box were used to study rearrangements at the surface before the first avalanche. They first showed that the size of rearrangements at the surface of the packing increases with the inclination angle. A 
few degrees before the avalanche starts, quasi-periodical large events are observed. These events, called 'precursors', consist of a large number of simultaneous moves of grains uniformly distributed over the surface of the packing.

Zaitsev et al [45] reported recently that the same kind of events occur in the bulk of a slowly inclined granular packing. These internal events were evidenced through nonlinear acoustical measurements by recording the acoustical signal component which is due to the self-nonlinearity of the granular material. These events were interpreted as quasiperiodic transient reorganizations of the weak-contact subnetwork occurring in the bulk of the packings. A recent work of Gibiat et al [14] confirmed the existence of precursors by recording sound pulses produced by rearrangements in the volume of the material. The latter experimental results agree qualitatively with those of computer simulations of inclined 2D packings [41] which evidence the occurrence of intermittent rearrangements of grain contacts in the bulk of the packings. Staron et al [41] investigated the evolution of the internal state of a 2D granular slope driven towards its stability limit, $\theta_{\mathrm{a}}$. They related precursors of avalanches to the intermittent mobilization of friction forces between the grains where the micro-structure undergoes an irreversible sharp modification during the inclination. These modifications of the granular micro-structure were assumed to result from the emergence of long-range correlations in the packing, but this conclusion needs to be further considered for 3D packings.

The previous numerical and experimental results show the growing susceptibility of the pile to avalanching when approaching the static angle of friction $\theta_{\mathrm{a}}$. However, beyond the observation of a metastable system approaching $\theta_{\mathrm{a}}$, and the experimental evidence of the existence of precursors to avalanches, neither the precise nature of these events occurring before the avalanche, nor the characteristics of the transition towards the avalanche are yet understood.

In this paper, we report the results of an experimental study of the dynamics of inclined 3D granular packings driven towards their stability limits. We characterize the dynamics of rearrangements at the surface of the packing and identify precursors of avalanches. Each precursor consists of a cooperative displacement of a large number of grains at the surface of the packing. This displacement, whose average amplitude is smaller than one grain diameter, does not change the mean slope of the pile in contrast to large slope changes which occur in avalanching piles. We show that the characteristics of these events depend significantly on the microscopic details of the interaction between grains and we discuss their physical origin. We then relate them to a macroscopic mobilization of friction of the packing with a stick-slip dynamics which confirms that long-range correlations develop at the approach of avalanche. In addition, we discuss the consequences of the emergence of a macroscopic correlation length on the packing stability. We show in particular that the avalanche angle, $\theta_{\mathrm{a}}$, depends strongly on the length of the packing.

\section{Experimental setup}

\subsection{Experimental device}

The experimental device used to incline a granular packing consists of a slowly rotating rectangular plexiglas box containing glass beads (see figure 1). The home-built system is 


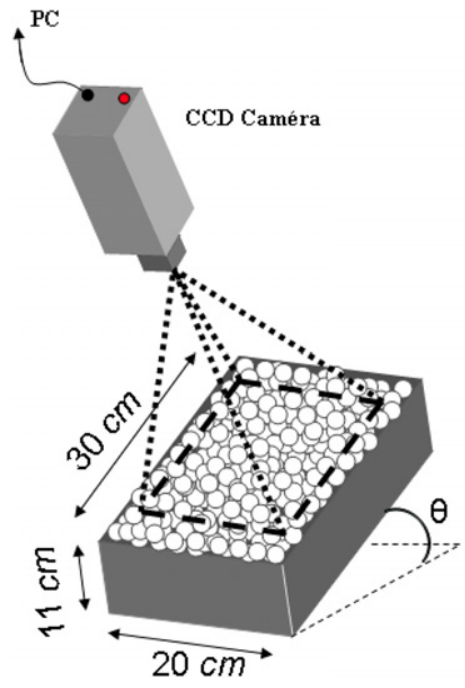

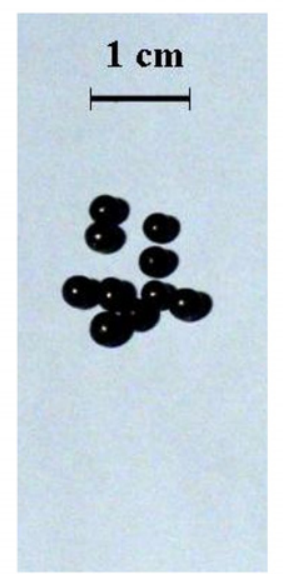

(a)

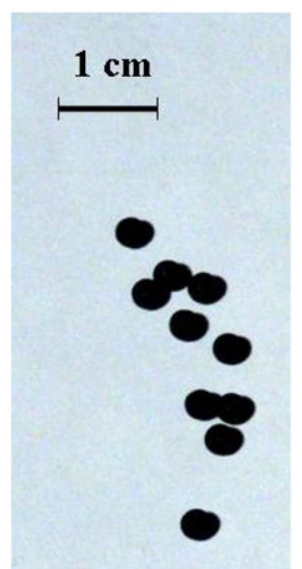

(b)

Figure 1. Experimental device. Left: sketch of the experimental setup. Right: photographs of the surface tinted beads (a) and volume tinted beads (b) used in the present experiments.

constituted of a heavy table and of a frame which can be inclined at different rates by means of a threaded stem linked to a stepping motor. The threaded stem is connected to the frame via a worm drive system. The rotation speed can be varied from $1^{\circ} \mathrm{min}^{-1}$ to $10^{\circ} \mathrm{min}^{-1}$. The box, which contains grains, and a camera used to scan the free surface are both fixed to the frame. The inclination angle is measured with an inclinometer also fixed to the frame. The precision of the angle measurement is $0.1^{\circ}$. This efficient experimental setup allows us to control the inclination operating speed and to decrease the effects of mechanical vibrations. For this, foam is used at the contact between the motor and the heavy table to damp vibrations from the motor. Moreover, the low rotation velocity protects the system from large noise levels. A calibration procedure is used to determine the relation between the rotation velocity and the motor voltage and to verify the uniformity of the rotation. It consists in plotting the inclination angle as a function of time and fitting it to a line.

The size of the box is $30 \mathrm{~cm} \times 20 \mathrm{~cm} \times 11 \mathrm{~cm}$ and the granular packing is constituted of glass beads of diameter $3 \mathrm{~mm} \pm 0.3$. To evaluate the sizes of the rearrangements at the surface of the packing during inclination, we used black beads since transparent beads produce light reflections which complicate the processing of images. We used two kinds of black beads, either volume tinted (type 1, figure 1(b)) or surface tinted (type 2, figure 1(a)). We evaluated the mean friction coefficient of each kind of bead by measuring the slip angle of a metal plate covered with beads on an inclined plane to which the same kind of beads was glued. We found $\left\langle\mu_{\mathrm{s} 1}\right\rangle=0.32 \pm 0.01$ and $\left\langle\mu_{\mathrm{s} 2}\right\rangle=0.34 \pm 0.01$ for beads of types 1 and 2 respectively. This small difference in the friction coefficient is due to the surface state which is different for the two kinds of beads because of the manufacturing process. The behaviour of a granular packing is strongly influenced by humidity. Therefore, during all our experiments the humidity and the temperature of the room were kept constant, with respective values of $50 \%$ and $T \approx 23^{\circ} \mathrm{C}$, thanks to 


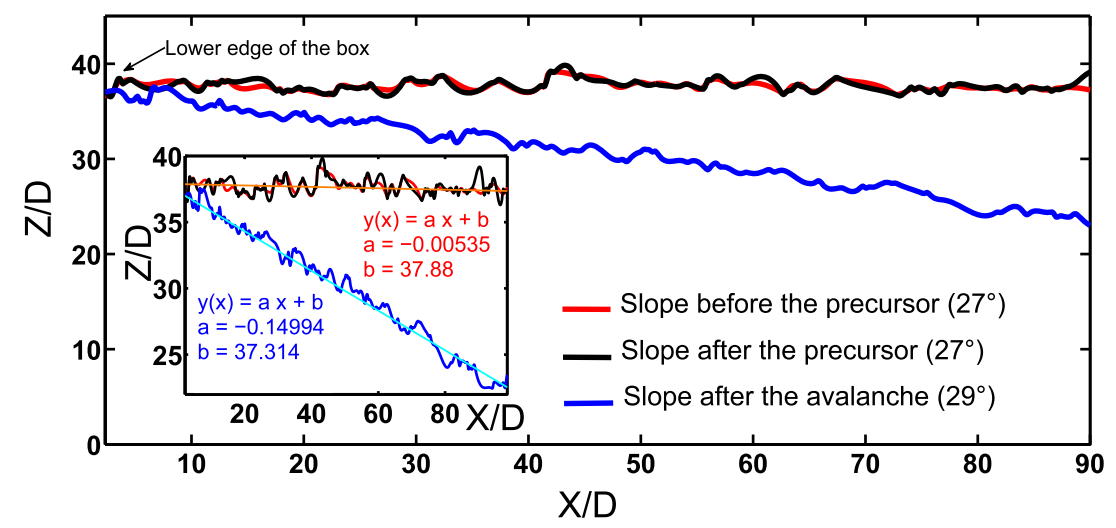

Figure 2. Slope of the pile before and after a precursor at $27^{\circ}$ (upper curves) and after the avalanche $\left(29^{\circ}\right.$, lower curve). $Z / D$ is the height of the pile normalized by the grain diameter and $X / D$ is the position along the box.

dehumidifiers and an air conditioner to reduce as far as possible the effects of capillarity and electrostatic forces.

\subsection{Experimental procedure}

In all experiments, the initial thickness of the packing is kept constant and equal to $8 \mathrm{~cm}$. The bottom of the box is prepared just once by gluing glass beads similar to those that constitute the piles onto a piece of wood. To ensure reproducible results, the same procedure is applied in all experiments for the preparation of the packing. First, a grid is put at the bottom of the box, then the box is filled up with glass beads and the height of the pack is made equal to a chosen value. This is achieved by eroding the free surface with a bar until it reaches the chosen height marked on the lateral walls of the box. Finally, the grid is removed from the box. Glass beads cross the grid and their movement ensures in that way the homogenization of the packing. Then, the height of the packing is adjusted once again as mentioned before. This procedure guarantees as far as possible the horizontality of the surface of the packing. By measuring the mass of beads which occupy the volume of the box, we estimated the mean packing fraction obtained by this procedure as $\langle\phi\rangle=0.59 \pm 0.01$. Finally, the packing prepared by the previous procedure is such that its height equals the height of the box. In that way, grains flow out of the box when an avalanche occurs. By contrast, grains remain in the box when small rearrangements or precursors occur and the slope of the pile does not change as shown in figure $2 .{ }^{3}$ The average height is 37.66 before the precursor and 37.71 after. The corresponding standard deviations are 0.57 and 0.51 respectively. The average surface remains thus parallel to the bottom surface as long as the inclination angle is less than the angle of avalanche.

Avalanches, which produce large surface changes, are not the focus of the present study. The inclination of the pile is therefore stopped after the occurrence of the first avalanche. This procedure is applied in all our experiments and establishes a quenched disorder in the initial state. As the initial structure of a granular packing is highly sensitive

${ }^{3}$ Preliminary experiments were performed before the start of the present study to evaluate the mean slope of the packing by measuring the height of the packing along a lateral vertical side of the box during inclination. 


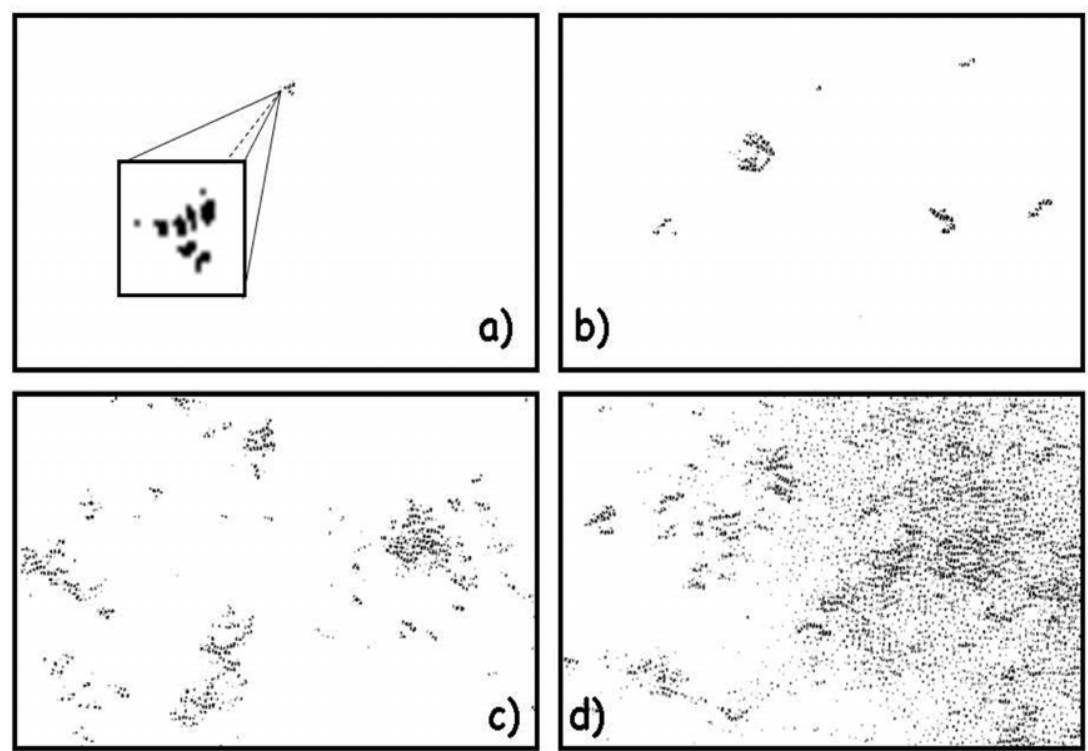

Figure 3. Some examples of rearrangements at the surface of the packing: (a) $3^{\circ}$; (b) $12^{\circ}$; (c) $20^{\circ}$; (d) $26^{\circ}$.

to its preparation, the dynamics of rearrangements and the occurrence of precursors at the surface of the packing depend on its history/preparation as shown by Kiesgen de Richter et al [20].

\subsection{Optical technique}

The images are processed in three steps. First, all the images of the movie recorded during the inclination process are extracted. In the images, the centres of grains appear as bright. Every image is subtracted from the next image. In that way, grains which have moved are evidenced. The elements of the 'difference' images correspond to the positions of the centres of grains before and after the rearrangements. Each rearrangement is related to the number of grains which move at the surface of the packing during the angular interval between two successive images. This interval is equal to about $0.01^{\circ}$ in our experiments, so events separated by an angular interval smaller than $0.01^{\circ}$ are considered to belong to the same rearrangement. We do not observe significant variations of our results when varying the angular interval provided that it is smaller than the characteristic gap of about $0.5^{\circ}$ between two successive large events. Then, the 'difference' images are binarized and the noise is reduced by applying a grey-level thresholding method. To recover the initial size of the rearrangements, we apply a dilation algorithm, followed by a hole-filling procedure. Finally, a particle analyser is used to extract the position, the area, and the eccentricity of clusters which correspond to rearranged zones. The conversion between pixels and glass bead diameter is obtained by calibration. The image analysis method described above allows us to evaluate the area fraction which is rearranged between two successive images. Figure 3 shows some examples of rearrangements which happen at the surface of the packing. Each subfigure presents rearrangements which occur at a different angle of inclination during the tilting. The size and number of rearrangements are seen to increase 
with the angle. Moreover, in our various experiments, rearrangements were observed to occur anywhere at the surface of the packing. There are no 'macroscopic heterogeneities' due to the initial preparation.

\section{Rearrangements at the surface of the packing}

\subsection{Evolution of the fraction of rearranged surface}

We describe below experimental results on the dynamics of superficial rearrangements during a single inclination of the packing from $0^{\circ}$ to the angle of avalanche $\theta_{\mathrm{a}}$. Figure 3 shows examples of events taking place at the surface of the packing during the inclination from $0^{\circ}$ to $\theta_{\mathrm{a}}$ for beads of type 2 (similar events were observed for beads of type 1 ). Each subfigure shows all rearrangements which occur between two consecutive images around the considered inclination angle. The number and average size of events are seen to increase with the inclination angle. While rearrangements are seen to be isolated from each other for small inclination angles, those which take place for angles larger than $25^{\circ}$ involve a large fraction of the grains located at the surface of the packing. Image analysis techniques, described in section 2.3, yield the surface fraction which has evolved between two consecutive images. Figure 4 shows the evolution of the rearranged surface fraction $S / S_{0}$ with the angle of inclination of the packing for independent experiments performed in similar conditions with beads of types 1 and 2 . Here, $S_{0}$ is the total area of the initial surface of the packing. The results are seen to be reproducible. The rearranged surface fraction exhibits an overall increase with the inclination angle.

For type 1 beads, a transition to an intermittent regime, in which large events occur almost periodically, occurs at about $20^{\circ}$. The latter events correspond to the large events called 'avalanche precursors' by Nerone et al [32]. Indeed, they are due to the simultaneous movement of a large fraction of grains at the surface of the packing in agreement with the findings in $[32,45]$ where it was observed that similar large events occur at angles smaller by a few degrees than the angle of avalanche.

Further, similar intermittent events were evidenced in numerical simulations of inclined 2D packings made in [41,40]. The results of nonlinear acoustical studies in [45] and those of the latter $2 \mathrm{D}$ simulations suggest that these large events are due to intermittent reorganizations of the network of weak contacts in the bulk of the packing. We detrended all signals $S / S_{0}$ and we applied a fast Fourier transform to them to determine the mean 'period' of the onset of precursors which was found to be $\langle\Delta \theta\rangle \approx 1.2^{\circ}$. Far from the critical angle, the behaviours of type 1 and 2 beads are similar. However, we observe differences near the critical angle. A single, large, precursor occurs reproducibly at $\theta \approx 26^{\circ}$ for surface tinted beads. The latter precursor consists of a cooperative displacement of the greatest part or the whole of the superficial grains. These grains belong to a small number of surface layers, typically one or two. The common amplitude of the displacement is small, being about one grain radius. This precursor does not change the average slope of the stacking. It differs qualitatively and quantitatively from the avalanche which occurs at a larger inclination angle, here about $30^{\circ}$ (figure 5 right). Therefore, the large macroscopic 'precursors', which take place close to the angle of avalanche, depend strongly on the microscopic details of the interactions at the grain scale. As concluded by Staron et al 

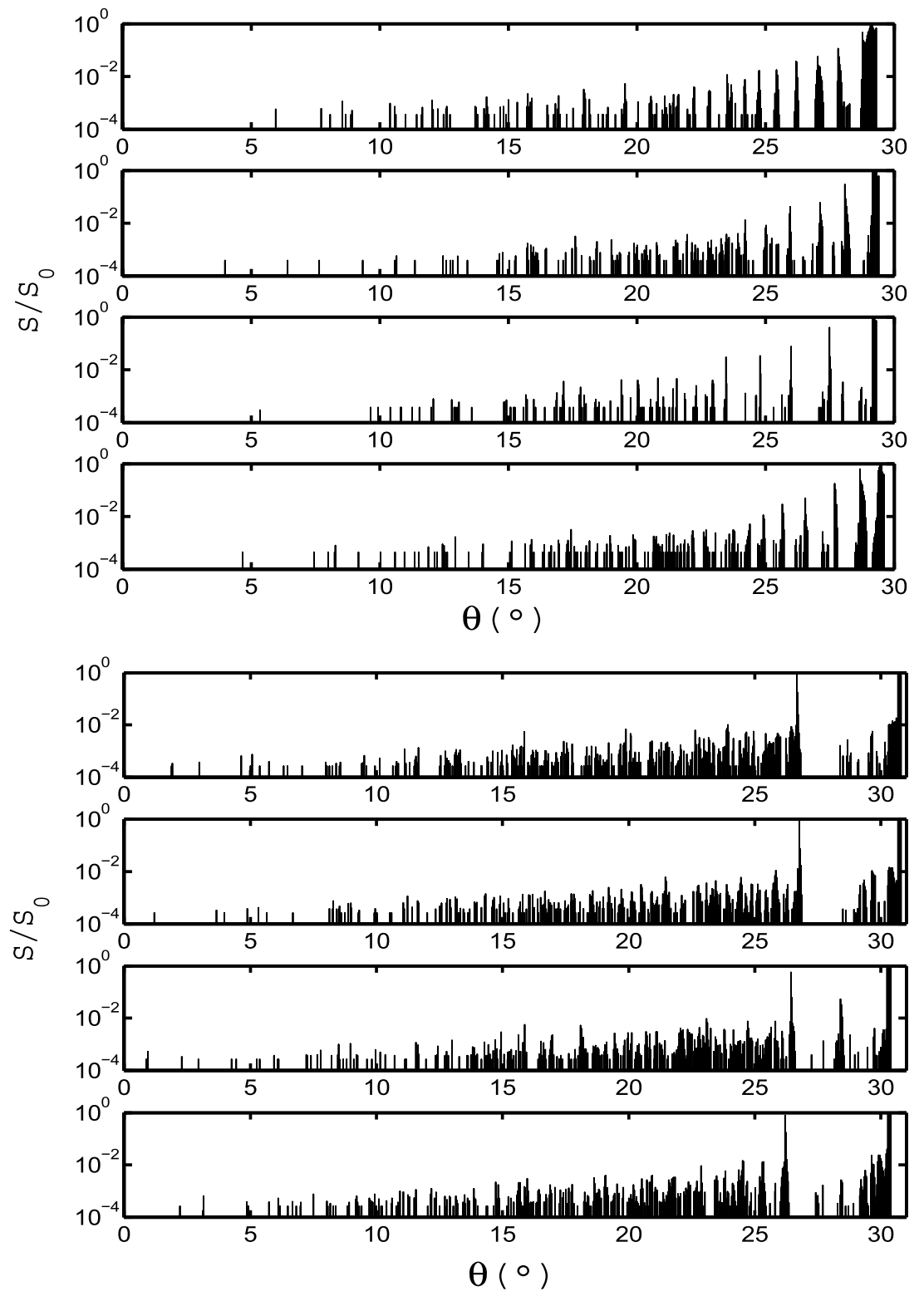

Figure 4. Some examples of signals $S / S_{0}$ for type 1 beads (top) and type 2 beads (bottom). All experiments are performed in identical conditions.

[40] from their 2D numerical simulations, precursors appear to result from a macroscopic mobilization of critical contacts controlled by a large scale correlation length.

\subsection{Evolution of the activity at the surface of the packing}

To characterize the dynamics and quantify the increase of the rearranged surface fraction, we present in figure 4 the activity of the packing during inclination, $A(\theta)$, defined as being the cumulative sum over the area of the rearranged surface normalized by $S_{0}$ :

$$
A(\theta)=\sum_{j=0}^{\lfloor\theta / \mathrm{d} \theta\rfloor} \frac{S(j \mathrm{~d} \theta)}{S_{0}} .
$$



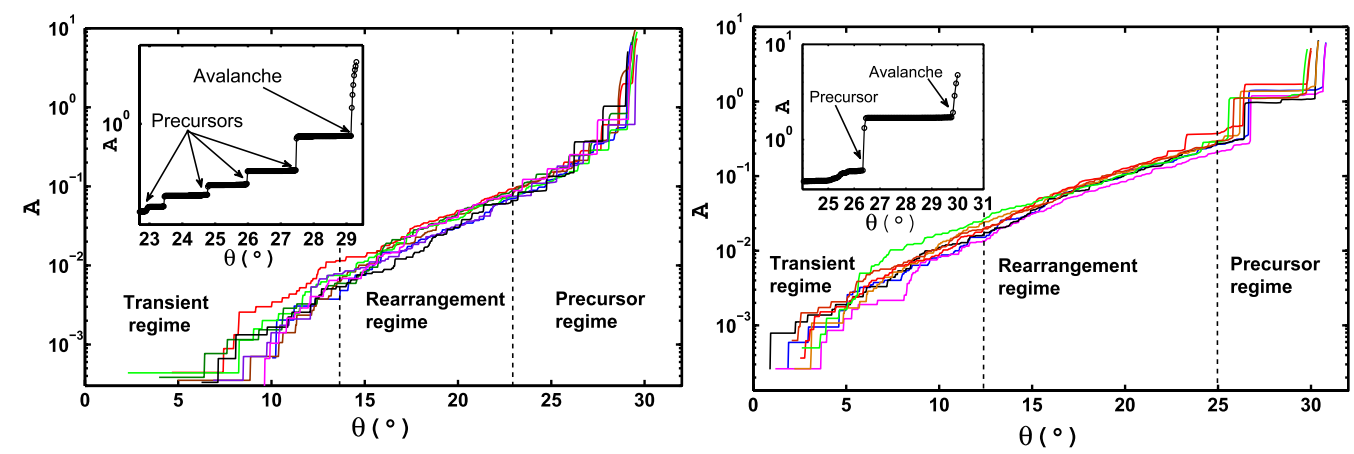

Figure 5. Evolution of the activity of the packing, $A(\theta)$, for type 1 beads (left) and type 2 beads (right). The insets are zooms on the precursor zone.

In relation (1), $\theta$ is the angle of inclination of the packing and $\mathrm{d} \theta$ is the angular interval between two consecutive images.

Results found for independent experiments performed in similar conditions are plotted in figure 5 to emphasize their very good reproducibility. Three regimes are evidenced in the evolution of the activity of the packing during inclination. First, for small angles, a transient regime, which depends significantly on the initial preparation of the packing, where some beads initially in metastable positions on the surface move. Second, a growth regime characterized by an exponential increase of the activity, where rearrangements involving a small number of beads occur. Third, a 'precursor regime', with pseudo-periodic large events, appears close to the critical angle. Last, the system avalanches. The most intense precursors take place for angles larger than $25^{\circ}$, but the first, less intense, ones are more difficult to detect. The minimum value of the angle at which they can be detected is of the order of $20^{\circ}$ and varies from experiment to experiment (see the inset of figure 5 left).

We point out that the growth of the activity in the two first regimes seems to be independent of the type of beads in contrast to the third one which depends significantly on the bead type (compare figure 4 left and figure 4 right). This observation can be interpreted by considering precursor events as a mobilization of friction through sliding of a large number of grains at the surface of the packing, a process which depends on the microscopic details at the grain scale. By contrast, the intermediate regime consists of localized destabilizations of grains at the surface which cause rearrangements of their neighbours which are in turn destabilized. This mechanism involves many degrees of freedom of grains and is thus relatively insensitive to the microscopic details of the interactions.

\section{Transition from the regime of small rearrangements to the precursor regime}

\subsection{Distribution of event sizes}

The dynamics of superficial rearrangements is also characterized by the distribution of event sizes during inclination and by the angular evolution of this distribution. The size of an event is defined as the area of the whole surface rearranged during this event. A rearrangement of surface $S$ which happens at an angle $\theta$ is composed of "clusters of 


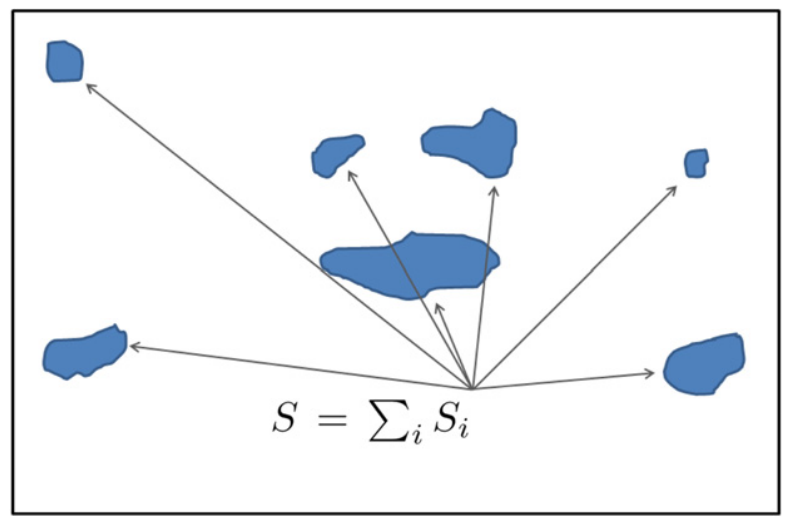

Figure 6. Decomposition of an event of size $S$ into a sum of $i$ clusters of size $S_{i}$.
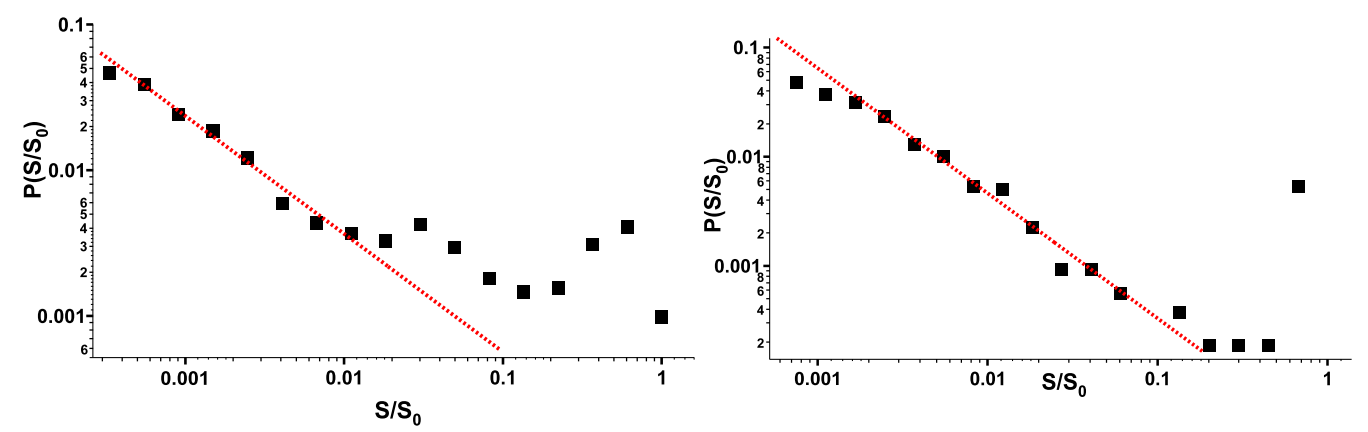

Figure 7. Log- $\log$ plot of the distributions of event sizes obtained from sets of 60 independent experiments: left for type 1 beads, right for type 2 beads. In the power law regime, the distributions are fitted by $\left(S / S_{0}\right)^{-\mu}$ with $\mu$ equal to 0.9 and 1.2 for beads of types 1 and 2 respectively.

rearrangements'. Each cluster has an area $S_{i}$ as shown in figure 6 . The fraction of rearranged area is equal to $S / S_{0}$ for the whole set of rearrangements and to $S_{i} / S_{0}$ for individual clusters. Figure 7 presents the distribution of $S / S_{0}$ for all events (and for all angles) for type 1 and type 2 beads. The displayed distributions are obtained from sets of 60 experiments. For type 1 beads, the distribution shows two regimes.

For small amplitude events the distribution decreases as a power law, $P\left(S / S_{0}\right) \approx$ $\left(S / S_{0}\right)^{-\mu}$ with $\mu=0.89$. For large events $\left(\left(S / S_{0}\right)>0.01\right)$, the distribution deviates from a power law and a peak appears for events whose size is approximately equal to $S_{0}$. A similar behaviour is obtained for type 2 beads with $P\left(S / S_{0}\right) \approx\left(S / S_{0}\right)^{-\mu}$ with $\mu=1.2$. For these beads, the power law decay still holds for large events since only a single large precursor happens (figure 4 right). Its size is of the order of the initial surface area of the packing. These results show us that the distribution departs from a power law behaviour when the precursor regime is reached, with an excess of large events in this regime. This is confirmed by a plot of the distribution of cluster sizes for type 1 beads, $P\left(S_{i} / S_{0}\right)$ (figure 8).

A clear power law decay is also observed for small clusters $P\left(S_{i} / S_{0}\right) \approx\left(S_{i} / S_{0}\right)^{-\mu}$ with $\mu=1.3$. This universal behaviour vanishes and a cut-off appears when the size of events reaches a few per cent of the total surface area $S_{0}$. These results suggest that the physical 


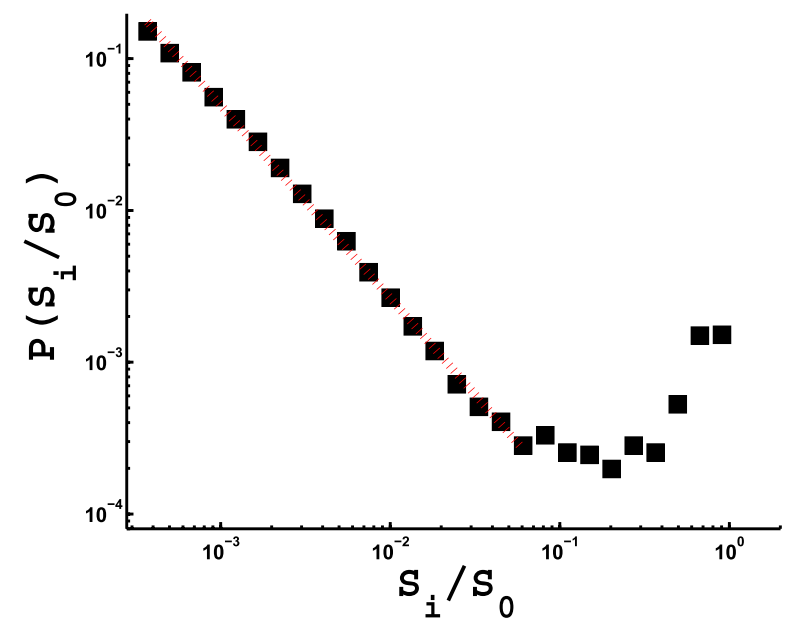

Figure 8. $\log -\log$ plot of the distribution of cluster sizes $S_{i}$ for type 1 beads. The line is a power law fit $\left(S_{i} / S_{0}\right)^{-\mu}$ with $\mu=1.27$.

mechanisms at the origin of small events differ from those at the origin of larger ones. These experimental observations are consistent with previous numerical results obtained by Prado and Olami [37] on the breaking of self-organized criticality in sandpile cellularautomata models. The latter authors attributed this breaking to a dominance of large events due to a 'snowball effect' which occurs at the surface of the packing when inertia effects are taken into account. We will show in section 6 that the characteristics of the precursors we observe in our experiments are inconsistent with this hypothesis but that they agree instead with the existence of successive slidings at the surface of the packing.

\subsection{Determination of the transition point}

To identify the transition point from the regime of limited rearrangements to the precursor regime, we plot in figure 9 the distribution of the sizes of events which take place in disjoint angular intervals of a constant width of $3^{\circ}$ when the origin of the interval ranges from $0^{\circ}$ to $\theta_{\mathrm{a}}$. The distributions are well approximated by a power law in their respective size ranges, for angles smaller than $18^{\circ}$. The transition point appears to be statistically located between $18^{\circ}$ and $21^{\circ}$, a result that cannot be obtained solely from the evolution of $S / S_{0}$ (see figure 4) with the inclination angle. Our results confirm that the latter transition occurs at about $20^{\circ}$ for type 1 beads with the appearance of large events whose size distribution differs from those of small rearrangements.

In contrast to results obtained in [8] on the statistics of the rearrangement sizes, the system does not show a self-organized criticality behaviour. This difference comes from the intrinsic non-stationary nature of our experiments where the applied constraint changes with time.

\section{Characterization of the regime of small rearrangements}

\subsection{Mean field trap model}

To describe the evolution of the rate of rearrangements in the regime of small rearrangements, we fitted the activity of the packing to a function $A_{\text {theo }}(\theta)$ similar to 


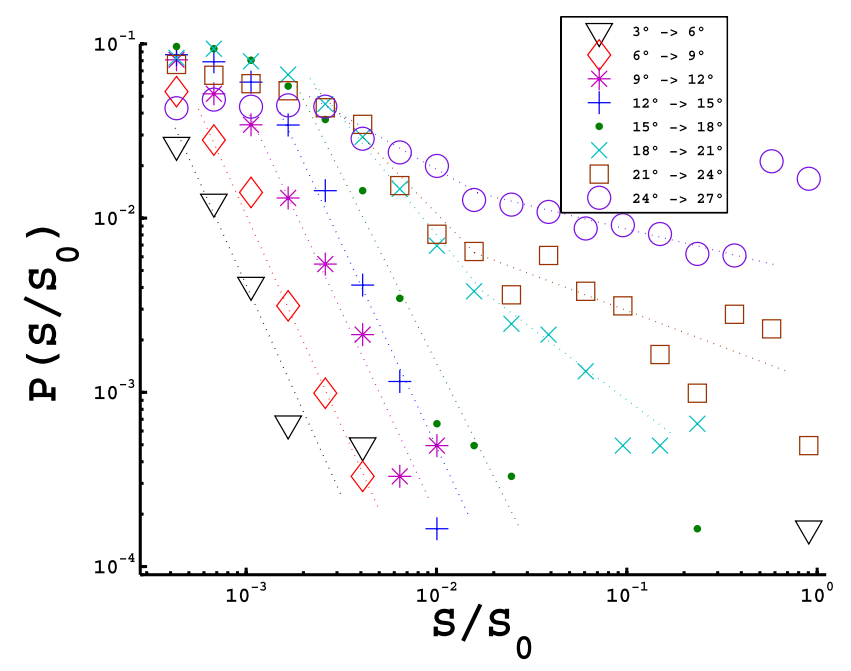

Figure 9. Evolution of the distribution of sizes of events, $P\left(S / S_{0}\right)$, occurring in a window of constant width $\left(3^{\circ}\right)$ with the inclination angle. The lines are guides for the eye.

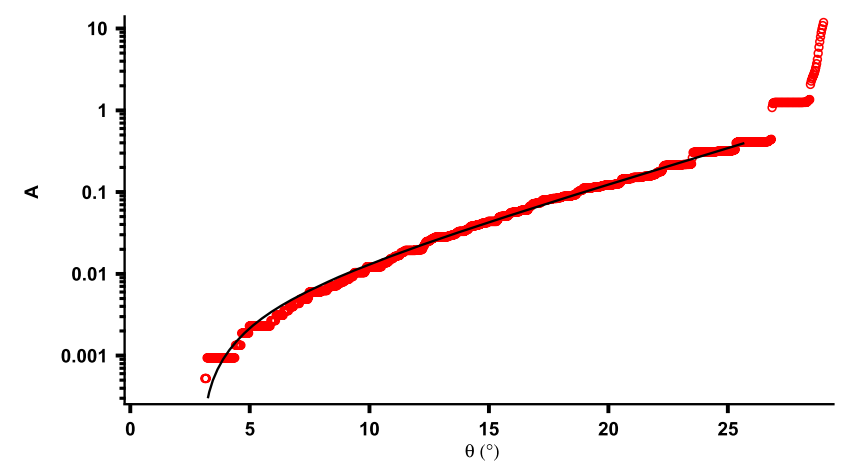

Figure 10. Fit of the type 1 bead activity to equation (2) which gives $\beta=0.20$ and $\theta_{0}=2.89$.

a function previously used by Nerone et al [32]. The latter function was obtained by considering the fluctuations of the activity due to precursors whose size grows exponentially with the inclination angle. Summing all contributions up to a given angle, Nerone et al [32] proposed

$$
A_{\text {theo }}(\theta)=\frac{K \mathrm{e}^{\beta \theta_{0}}}{\beta}\left(\mathrm{e}^{\beta\left(\theta-\theta_{0}\right)}-1\right)
$$

where $\beta$ is a parameter which describes the rearrangement rate, and $\theta_{0}$ is the angle at which the first rearrangement takes place.

Figure 10 shows that the activity for beads of type 1 is well fitted by equation (2). In contrast to [32], we observe an increase of the activity when the inclination angle comes close to the critical angle $\theta_{\mathrm{a}}$. This speedup of the dynamics is related to the increase of the mean size of events in the precursor regime.

In the following, phenomenological arguments derived from Bouchaud's model $[6,26]$ are given to justify the choice of the function (2) to describe the evolution of the system 
in the small rearrangement regime. Bouchaud's model describes the thermal dynamics of an ensemble of independent particles assumed to be in a space of traps characterized by a given probability density function $\rho(E)$ for a depth of traps $E$. The state of the material is described by the probability density $P(E, t)$ to be in a trap of depth $E$ at time $t$ and the dynamics is controlled by the rate per unit time $\Gamma_{0} \exp (-\beta E)$ at which a particle escapes from its trap due to thermal agitation at a temperature $1 / \beta$. Relying on a trap model, each bead at the surface of the packing is considered to be blocked in a trap whose characteristics are determined by the positions of its neighbours. Thus, its stability is determined by a local friction coefficient and by the local geometry. When an external perturbation (or an internal one created by the system itself) acts, the bead can start to move if the perturbation is strong enough.

We define a mean effective friction coefficient, $\mu_{\mathrm{s}}^{*}$, which is such that $\tan ^{-1}\left(\mu_{\mathrm{s}}^{*}\right)$ represents the mean depth of traps at the surface of the packing in the as-prepared state $(\theta$ $=0)$. The inclination process is modelled by a continuous decrease of the trap depth $h(\theta)$, $h(\theta)=\arctan \left(\mu_{\mathrm{s}}^{*}\right)-\theta$, and we suppose that the dynamics is controlled by an activation process. The probability to escape from the trap, $P_{s}(\theta)$, must increase with $\theta$, and we assume that $P_{s}(\theta)=C \mathrm{e}^{-\beta(h(\theta))}$, where $C$ is a constant of normalization, and $\beta$, that we suppose to be constant, represents the mean level of perturbations created by bead motion and by the external noise ${ }^{4}$. With this hypothesis and the additional assumption that particles are independent, the fraction of rearranged surface at $\theta,\left(S / S_{0}\right)(\theta)$ is proportional to the fraction of beads which move at this angle, $N(\theta) / N: S / S_{0} \approx N(\theta) / N=C \mathrm{e}^{-\beta(h(\theta))}$ Finally, the activity of the packing can be approximated by

$$
A(\theta)=\int_{\theta_{0}}^{\theta} \frac{S(u)}{S_{0}} \mathrm{~d} u=C \frac{\mathrm{e}^{-\beta \operatorname{atan}\left(\mu_{\mathrm{s}}^{*}\right)} \mathrm{e}^{\beta \theta_{0}}}{\beta}\left(\mathrm{e}^{\beta\left(\theta-\theta_{0}\right)}-1\right) .
$$

Equation (3) is identical to equation (2) with $K=C \mathrm{e}^{-\beta \operatorname{atan}\left(\mu_{\mathrm{s}}^{*}\right)}$. The evolution of the activity is exponential with a growth rate controlled by $\beta$. The derivation of equation (3) does not rely on premises on precursors in contrast to the assumptions used in [32] to obtain equation (2). The eventual influence of precursors on the exponential behaviour of the activity is further discussed in section 5.2 and in section 6.1 where noise is applied to the packing during inclination. The precursors are shown to be suppressed when the noise intensity is high enough, while the exponential behaviour is left unchanged.

\subsection{An OFC type cellular automaton}

Olami, Feder and Christensen (OFC) [34] mapped the Burridge-Knopoff spring-block model of earthquakes into a cellular automaton on a square lattice. In their model, a stress variable, initially chosen at random between 0 and a threshold $s_{M}$, is assigned to each site. Stresses are increased simultaneously and uniformly over the lattice until the stress of a certain site is equal to or exceeds $s_{M}$. This event is the start of an earthquake during which the stress of this site evolves in the following way: a given fraction of it is redistributed in equal amounts to its four neighbours then this stress is set equal to zero. This redistribution rule can possibly create new unstable sites among the considered neighbours which evolve in turn according to the previous rule. This process continues

${ }^{4}$ A different point of view would be to consider $\beta$ as the standard deviation of the distribution of trap depths and $h(\theta)$ as the mean depth. Then, the 'noise' would represent the rugosity of the surface. 
until the stresses of all sites are less than $s_{M}$. Once all sites are relaxed, the simultaneous and uniform increase of stresses resumes. The OFC model is spatially homogeneous and displays self-organized criticality when the boundary conditions are open but not when they are periodic. Different kinds of heterogeneities have been included in extensions of the OFC model (see for instance [44] and references therein). Variants of it were introduced to capture general features of landslides [35] and of sandpiles [37,31].

Prado and Olami [37] proposed a model aiming to reproduce the behaviour of a sandpile built by dropping individual grains. The model includes inertia effects by taking into account the transfer of momentum during rearrangements at the surface of the pile. The model does not exhibit a self-organized behaviour, but shows instead a behaviour dominated by large events as observed experimentally [17]. Nerone and Gabbanelli [31] compared their experimental results on superficial rearrangements of slowly driven packings of four layers of grains with results of numerical simulations performed with an OFC type cellular automaton. Each site $k$ of a square lattice is characterized by its number of grains, which gives the local height of the packing, and by the momentum of a grain. When the lattice is progressively inclined, a given grain moves or not from site $k$ to site $j$ according to whether the slope associated with this move exceeds or not the critical value of site $k$. As in the model of Prado and Olami [37], the critical slope at site $k$ depends on the local momentum. A bead which moves gains a unit of momentum but loses part of its initial momentum which is transferred to layers of beads located below it. The critical slopes depend thus on the history of grain rearrangements through the evolution of momenta. Nerone and Gabbanelli [31] showed that the inertia effect does not significantly affect the dynamics of small events but plays an important role when an avalanche starts. They characterized the evolution of the activity (equation (1)) with the inclination angle. This activity exhibits an exponential increase as observed experimentally, but no precursor regime occurs before the avalanche angle.

To test further for the robustness of equation (2), we performed Monte Carlo simulations with a variation of the OFC model in which each site $(i j)$ of a square network, with $N=10000$ sites and periodic boundary conditions, is characterized by a local slope $p_{i j}(\theta)$ which depends on the inclination angle $\theta$. A critical site is a site whose slope is larger than a critical slope $\varphi_{c}$, fixed since the start and identical for all sites. The slope of a critical site is not reset to zero, as is the stress in the OFC model, but is updated according to equation (4), where $\alpha$ is a local relaxation coefficient $(\alpha<1)$. At the start, the $p_{i j}(\theta=0)$ are chosen according to a Gaussian distribution with a mean $\mu$ and a variance $\sigma^{2}$. The inclination process is simulated by an increase, at each iteration, of all $p_{i j}$ by a quantity $\mathrm{d} \theta$. Unstable sites $\left(i_{\mathrm{c}} j_{\mathrm{c}}\right)$, with $p_{i_{\mathrm{c}} j_{\mathrm{c}}}(\theta)>\varphi_{\mathrm{c}}$, are then identified. Finally, the system relaxes according to the rules given by equations (4)-(6):

$$
\begin{aligned}
& p_{i_{\mathrm{c}} j_{\mathrm{c}}}(\theta)=p_{i_{\mathrm{c}} j_{\mathrm{c}}}(\theta)-\alpha \varphi_{\mathrm{c}} \\
& p_{i_{\mathrm{c}} j_{\mathrm{c} \pm 1}}(\theta)=p_{i_{\mathrm{c}} j_{\mathrm{c} \pm 1}}(\theta)+\frac{\alpha \varphi_{\mathrm{c}}}{4} \\
& p_{i_{\mathrm{c} \pm 1} j_{\mathrm{c}}}(\theta)=p_{i_{\mathrm{c}} j_{\mathrm{c} \pm 1}}(\theta)+\frac{\alpha \varphi_{\mathrm{c}}}{4} .
\end{aligned}
$$

In contrast to the critical slope in the automaton of [31], $\varphi_{\mathrm{c}}$ does not depend on the past of sites. The packing history is fully contained in the distribution of slopes 

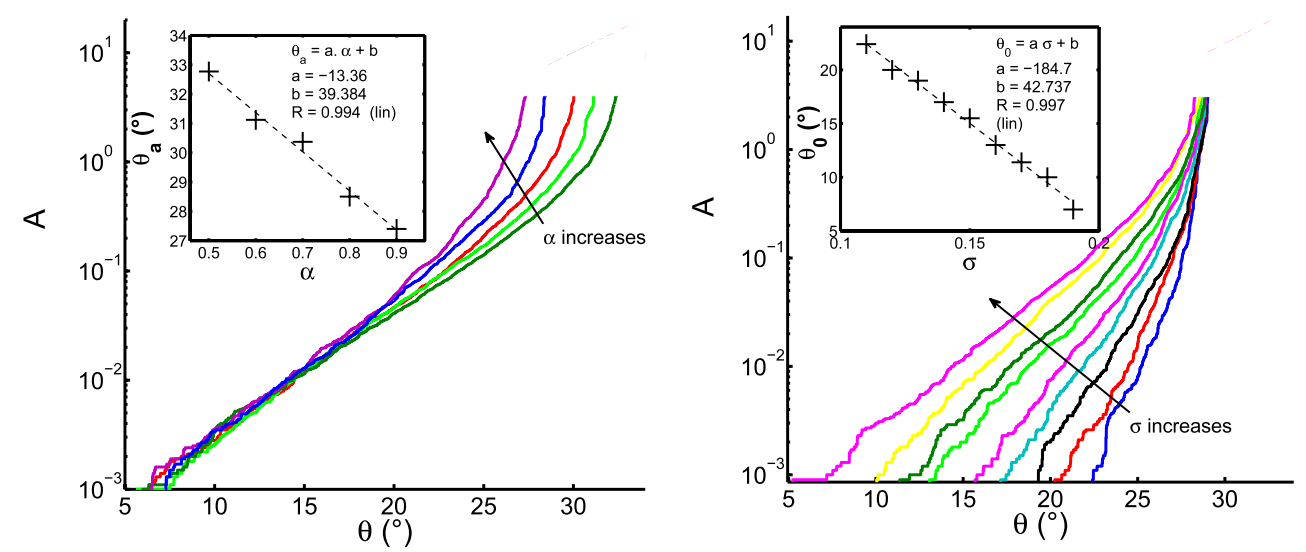

Figure 11. Left: simulated activity for various values of $\alpha$ as a function of $\theta$. Each curve is a realization obtained for $N=10000, \mu=0, \varphi_{\mathrm{c}}=0.6, \sigma=0.19$ and $\mathrm{d} \theta=10^{-4}$. The inset shows the evolution of the avalanche angle $\theta_{\mathrm{a}}$ as a function of $\alpha$. Right: simulated activity for various values of $\sigma$ as a function of $\theta$. Each curve is a realization obtained for $N=10000, \mu=0, \varphi_{\mathrm{c}}=0.6, \alpha=0.8$, and $\mathrm{d} \theta=10^{-4}$. The inset shows the evolution of $\theta_{0}$ as a function of $\sigma$.

for a given inclination angle. Further, inertia effects are neglected as the system is equilibrated instantaneously between two successive inclinations. The characteristic time of a rearrangement is indeed negligible as compared to the characteristic time of the increase $\mathrm{d} \theta$ of the inclination angle. The number of rearranged sites, $N_{\mathrm{r}}(\theta)$, is used to update a normalized cumulative sum which represents the activity, $A$, as defined previously. The simulation is stopped when the 'avalanche' takes place at an angle $\theta_{\mathrm{a}}$ for which the constraint can nowhere be satisfied. To compare the experimental and the simulated results, we have to evaluate three free parameters $\left(\alpha, \varphi_{\mathrm{c}}\right.$, and $\left.\sigma\right)$. The critical local slope, $\varphi_{\mathrm{c}}$, can be seen as the critical angle at which a bead supported on a triangle of three contacting beads loses its equilibrium. This angle was obtained to be about $35^{\circ}$ in [2]. In the following, we fix therefore the value of $\varphi_{c}$ at $\varphi_{c}=0.6$. To evaluate the remaining parameters, we plot first in figure 11 the activity of the packing for different values of $\alpha$ at fixed $\sigma$ (left) and for different values of $\sigma$ at fixed $\alpha$ (right). As observed in [31], the activity increases exponentially until it diverges close to the critical angle and no precursor regime is evidenced. Figure 11 (left) shows that at fixed $\sigma, \theta_{\text {a }}$ decreases linearly with $\alpha$. By contrast, at fixed $\alpha, \theta_{\mathrm{a}}$ keeps constant for different values of $\sigma$ but the value of $\theta_{0}$ decreases linearly with $\sigma$. Thus, $\sigma$ and $\alpha$ have separate effects on the activity of the packing. The parameter $\alpha$ can then be chosen first from the experimental avalanche angle $\theta_{\mathrm{a}}$ which is close to $29^{\circ}$ (figure 5) as $\alpha=0.8$. Then $\sigma=0.19$ is obtained from the experimental value of the angle $\theta_{0}$, about $5^{\circ}$ (figure 5 ), at which the first rearrangements occur. Figure 12 shows a typical evolution of the simulated activity as a function of the inclination angle $\theta$ for the indicated values of the parameters $(\alpha=0.8, \sigma=0.19$, $\left.\varphi_{\mathrm{c}}=0.6\right)$. The simulated activity is compared to the experimental activity for type 1 beads in figure 12 and is fitted to equation $(3)^{5}$. There is a reasonable agreement between

5 The simulated activity is fitted to equation (3) only between $10^{\circ}$ and $25^{\circ}$ to avoid the transient regime where the dynamics is not established. 


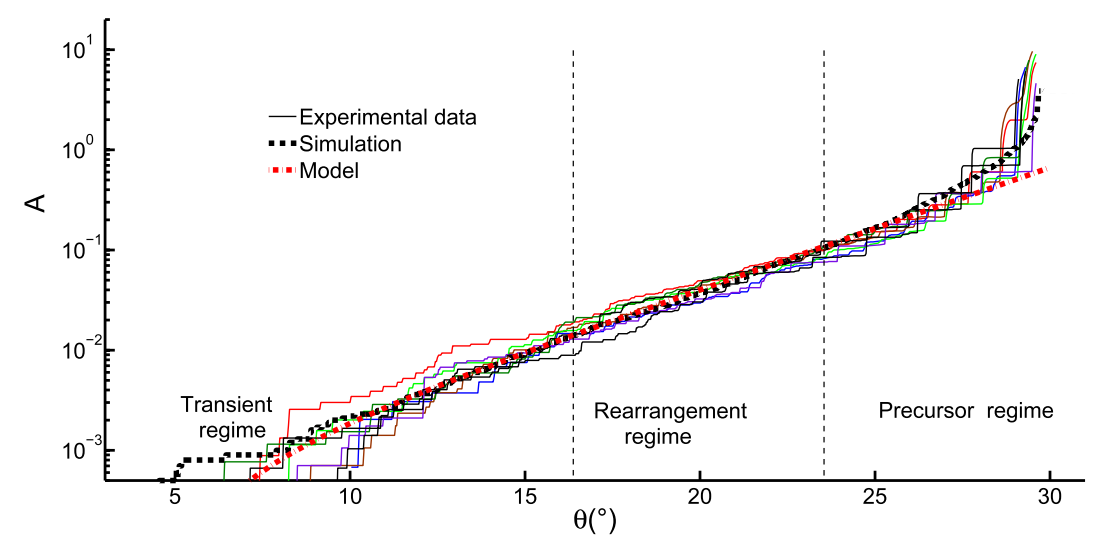

Figure 12. Evolution of the experimental and simulated activities during an inclination for $N=10000, \alpha=0.8, \varphi_{\mathrm{c}}=0.6, \sigma=0.19, \mu=0$ and $\mathrm{d} \theta=10^{-4}$. The simulated activity is fitted to equation $(3)\left(\beta=0.26, \theta_{0}=5.3^{\circ}\right)$.

the simulated, the calculated and the experimental activities in spite of the scattering of the latter. Equation (3) accounts fairly well both for the angular dependence of the experimental activity (figure 10) and for that of the simulated activity for angles well below the critical angle $\theta_{a}$ (figure 12$)^{6}$.

The growth rate of the activity in the rearrangement regime appears to be, in essence, independent of the microscopic details of the interactions between grains in contrast to what is observed in the precursor regime. The simulation results suggest that the exponential behaviour might be unrelated to the existence of a precursor regime. This agrees with the experimental results of section 6.1 which show that noise applied to the packing during inclination tends to suppress precursors when its intensity is high enough while it leaves the exponential behaviour unchanged. The exponential behaviour presents universal features imposed by the necessity for the system to fulfil constraints imposed by gravity. Kiesgen de Richter et al [20] studied the influence of the history of an inclined packing on its activity by applying a series of consecutive forth-and-back tilting cycles to the grain container. During a cycle, the box is first inclined from the zero angle to a maximum angle $\theta_{m}$ chosen to be close to the critical angle and then it is inclined back to the zero angle. The activity at the surface of the packing is followed during the forth-tilting parts of successive cycles (figure 6 of [20]). The overall activity at a given inclination angle $0<\theta \leq \theta_{\mathrm{m}}$ decreases significantly during the first three cycles. The angle of occurrence of the first rearrangement increases concomitantly. After three cycles, the surface enters a rather stable, aged, configuration which is kept when the packing is further inclined between 0 and $\theta_{\mathrm{m}}$. The angle at which the activity resumes after an ageing cycle between 0 and $\theta_{\mathrm{m}}$ is supposed to be controlled by the fluctuation of the distribution of the local slopes. The latter effect is simply observed with the automaton described above. Figure 13 presents the evolution of the distribution of local slopes during an inclination for an initial standard deviation, $\sigma$, equal to 0.2 . The mean local slope increases during the inclination and the standard deviation tends to decrease with $\theta$ as shown in the inset of figure 13 .

6 The results of a more detailed study of the influence of the various parameters on the dynamics of the OFC automaton will be presented in a future publication. 


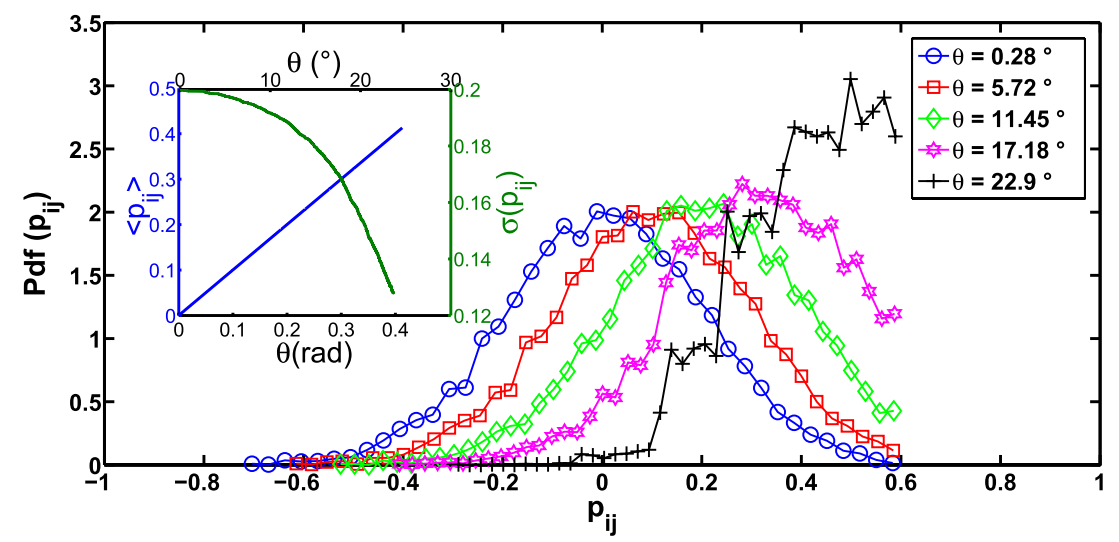

Figure 13. Evolution of the distribution of the local slopes $p_{i j}$ during an inclination for $N=10000, \alpha=0.8, \varphi_{\mathrm{c}}=0.6, \sigma=0.2, \mu=0$ and $\mathrm{d} \theta=10^{-4}$. The inset shows the evolution of the mean and the standard deviation of the distribution with the inclination angle $\theta$.

The linear evolution of the mean local slope results from equations (4)-(6) which describe how the local slope of a critical site and those of its 'four' neighbours are updated. The sum of these five local slopes remains the same before and after this updating. The sum of all slopes, and thus the mean local slope, remains unchanged once all updatings have been performed for the considered angle $\theta$. Then $\theta$ is increased by $\delta \theta$, that is all local slopes are increased by $\delta \theta$ and updating resumes. Consequently, the average local slope at a given $\theta$ is simply equal to $\theta$ because this average is zero for $\theta=0$. As expected, the simulation results in the inset of figure 13 show that the average local slope increases linearly with $\theta$ with a slope of 1 . Tilting the granular packing affects the fluctuation of local slopes at the surface and is at least partially responsible for the ageing effects reported in [20]. Figure 11 (right) shows the activity as a function of the inclination angle for different values of $\sigma$. The angle of the first rearrangement increases when $\sigma$ decreases. The latter result also comes from the fact that the critical angle is not significantly modified when $\sigma$ changes (figure 11).

\section{Characterization of the precursor regime}

In this section, we identify parameters which influence the emergence of precursors and we describe some of their characteristics.

\subsection{Influence of noise on precursors}

The influence of noise on precursors is qualitatively studied thanks to a vibrating device installed on the experimental setup.

The vibration level created via periodic pulses is evaluated with an accelerometer fixed to the box which contains grains. Figure 14 shows the evolution of $S / S_{0}$ and $A$ for different values of the acceleration applied to the box obtained for pulse frequencies equal to $50 \mathrm{~Hz}$. Increase of the noise intensity tends to suppress precursors. Discontinuities in the system evolution are less and less frequent and the intermittent behaviour of the system near the 

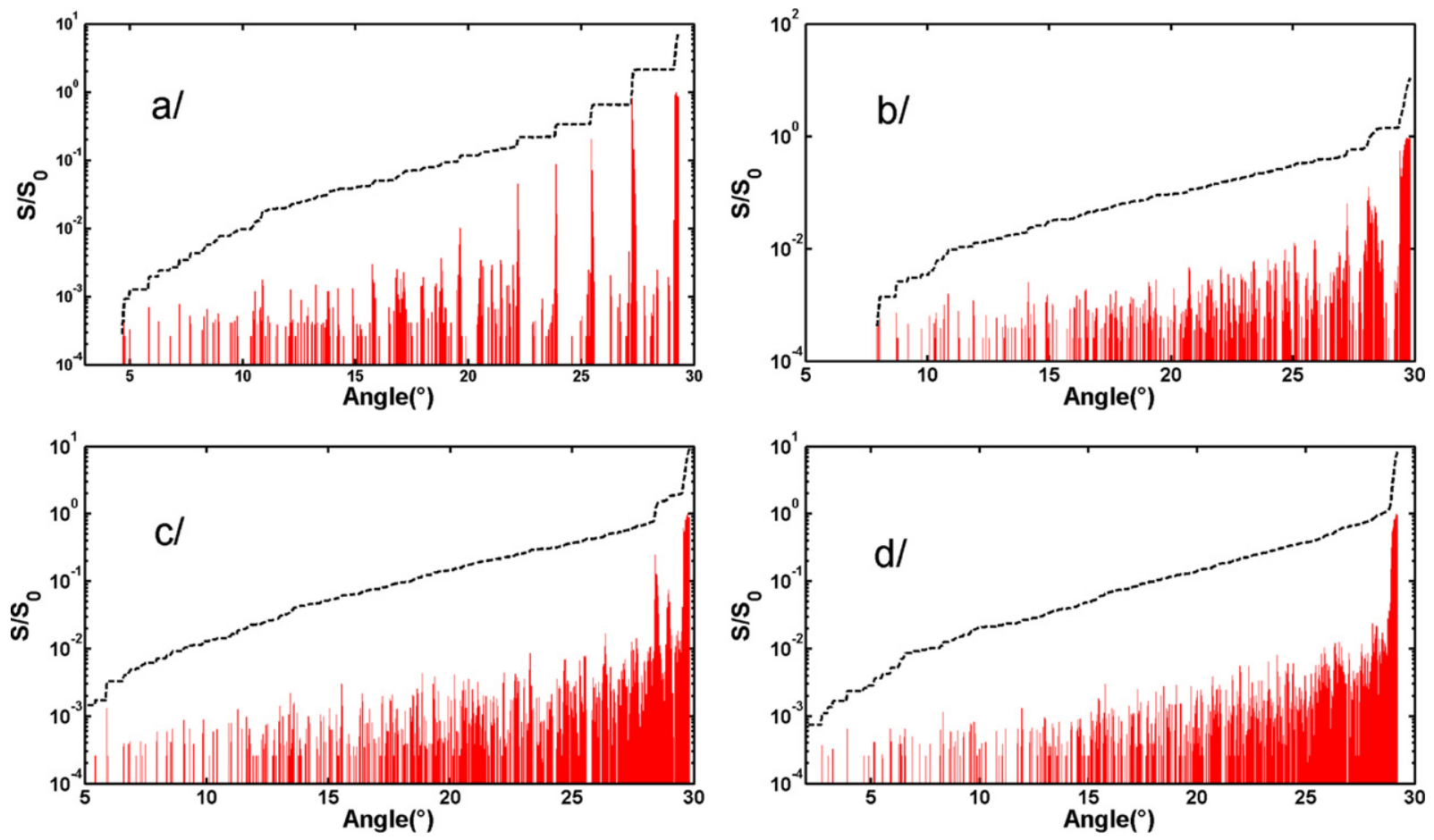

Figure 14. Influence of noise level on precursors as shown by four experiments in which the noise level increases from $a$ to $d$. The maximal accelerations are equal to (a) $0.05 \mathrm{~m} \mathrm{~s}^{-2}$, (b) $0.20 \mathrm{~m} \mathrm{~s}^{-2}$, (c) $0.25 \mathrm{~m} \mathrm{~s}^{-2}$ and (d) $0.40 \mathrm{~m} \mathrm{~s}^{-2}$.

critical angle disappears. Figure 14 shows further that the activity depends exponentially on the angle in a wide angular range even when the level of noise is high. We conclude that precursors are significantly sensitive to mechanical perturbations. We have verified this sensitivity in the $10-50 \mathrm{~Hz}$ frequency range.

\subsection{Influence of the initial preparation on precursors}

Because of the difficulty in precisely controlling the packing fraction of a large packing, we modified the preparation method to study the role of the initial packing fraction. With the volume of the box filled with beads being kept constant, the mass of the packing was measured to evaluate the packing fraction. When the packing was prepared with the method described in section 2.2, the packing fraction is found to be $\phi=0.594 \pm 0.01$. A different method, which consists in a step-by-step box-filling, was then used to change significantly the packing fraction. A few layers $(\approx 2)$ of grains were deposited in the box, then a weight was put on them to densify the packing and the process was repeated until the box was filled. By measuring the mass of grains in the box, we evaluated the packing fraction as $\phi=0.64 \pm 0.01$. Figure 15 shows the evolution of the mean activity, obtained from five independent experiments, for both packing fractions. The activity of the dense packing is smaller than the activity of the loose packing. The rearrangement regime starts at a larger angle for the dense packing than it does for the loose packing. One reason is that the surface of the packing is less rough in the dense case. Despite these differences, the packing evolution is quite similar in both cases with an exponentially 


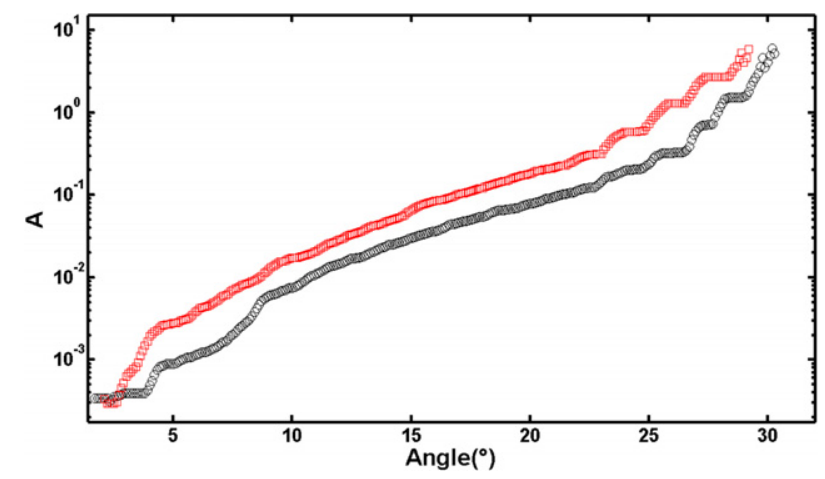

Figure 15. Influence of the initial packing fraction on the dynamics for $\phi=0.594$ (red squares) and $\phi=0.64$ (black circles). Each curve is a mean over five independent realizations.

varying activity in the small rearrangement regime and the existence of precursors which appear approximately at the same angle. Therefore, the packing fraction is concluded not to be a critical parameter for the occurrence of precursors.

\subsection{Displacements during successive precursors}

To evaluate the displacement field at the surface of the packing due to precursors, we compared the bead positions (their centres are bright) before and after each precursor with a particle tracking algorithm. With this method, displacements of grains which are smaller than a grain diameter can be measured for each precursor. This condition is fulfilled by all precursors observed in our experimental conditions. Figure 16 (top) presents an example of a displacement field measured for a precursor which appeared at $25^{\circ}$. All grains at the surface of the packing are seen to move but their displacements depend on their positions along the length of the box (horizontal axis). Grains which were initially at the top (right part) of the box undergo larger displacements that those which were initially at the bottom (left part). A large finite size effect thus exists along the length of the box up to $x / D \approx 50$ (the box is $30 \mathrm{~cm}$ long, that is about $100 D$ for beads of $3 \mathrm{~mm}$ diameter). Preliminary experiments were performed (see the appendix) to study the influence of finite size effects on the avalanche angle. Figure 17 shows examples of the total displacement of beads, $\delta$, expressed in units of bead diameter during a precursor as a function of the initial position of beads along the length of the box. In figure 17, the last four precursors which take place before the avalanche are characterized in this way. The abscissa of each vertical set of six points gives the angle at which the corresponding precursor appeared. Each curve is fitted to an exponential, $\delta(\theta)=a \mathrm{e}^{b \theta}$. The slopes of all lines are essentially the same and their mean value is $\langle b\rangle=\frac{1}{3}$. The displacements of grains during precursors increase thus exponentially during the non-stationary process of inclination. Precursors correspond to a global mobilization of grains at the surface of the packing resulting from microdisplacements of the whole surface. These displacements vary exponentially and are strongly influenced by confinement by the upper part of the side of the inclined box located at $X / D=0$ (figure 16, left side) as suggested in [1] in the study of avalanches of rice piles. Since precursors appear quasi-periodically, the system 


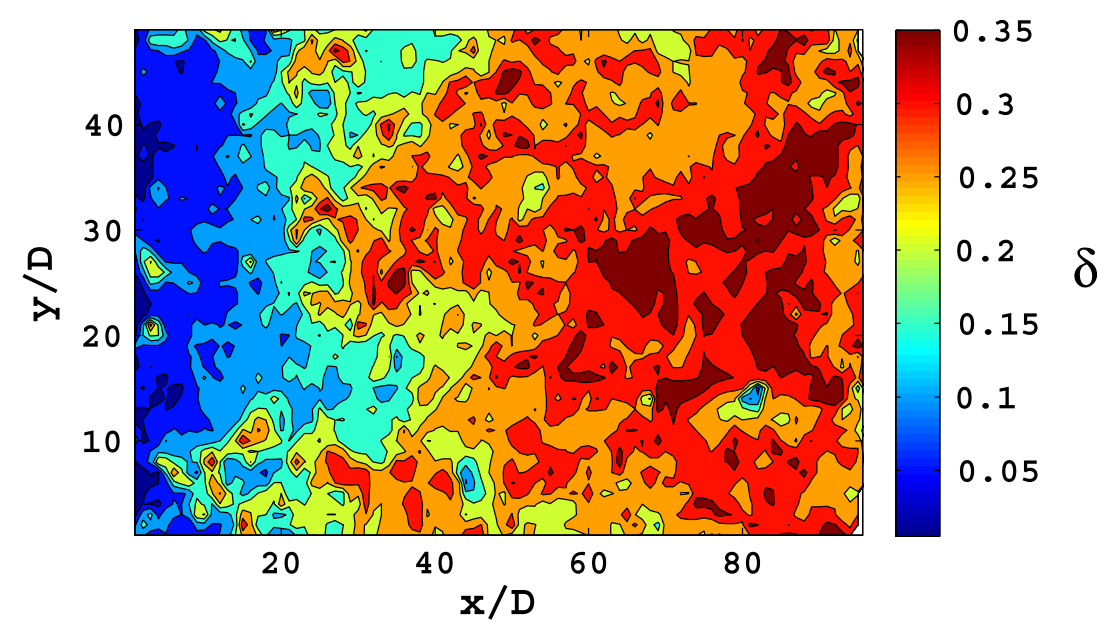

Figure 16. Total displacements of type 1 grains, $\delta$, at the surface of the packing. $\delta$ is expressed in grain diameter units. Grains move parallel to the surface of the packing.

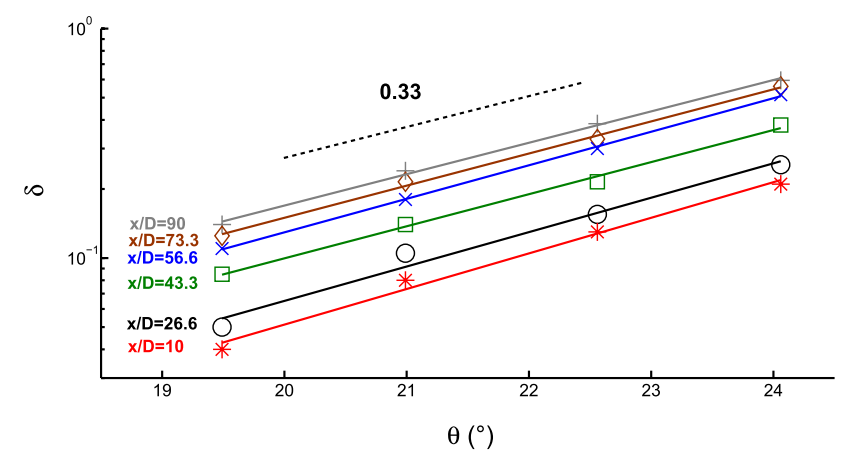

Figure 17. Total displacements of type 1 grains, $\delta$, during four consecutive precursors at the surface of the packing as a function of the inclination angle, and for various values of their position along the box length, $x / D$. $\delta$ is expressed in grain diameter units.

presents a non-stationary stick-slip type behaviour. As the displacements which occur during precursor events are observed to be smaller than one grain diameter (figure 17), we conclude, unlike Nerone and Gabbanelli [31], that inertia effects are not the relevant mechanisms at the origin of precursors as pointed out previously in section 4 .

\subsection{Stick-slip type behaviour of grains}

To acquire additional information on the grain displacements during a precursor, we used a high-speed camera. As they are fast during a precursor, the displacements of all grains at the surface cannot be followed with a sufficient temporal resolution. We chose then to zoom at the centre of the packing surface and to restrict the study to the displacements of a few type 1 grains (30 grains). From numerical calculation of the time derivatives of their positions, we deduced the temporal evolution of the grain velocity during a precursor which appeared at $24^{\circ}$ (see figure 18). 

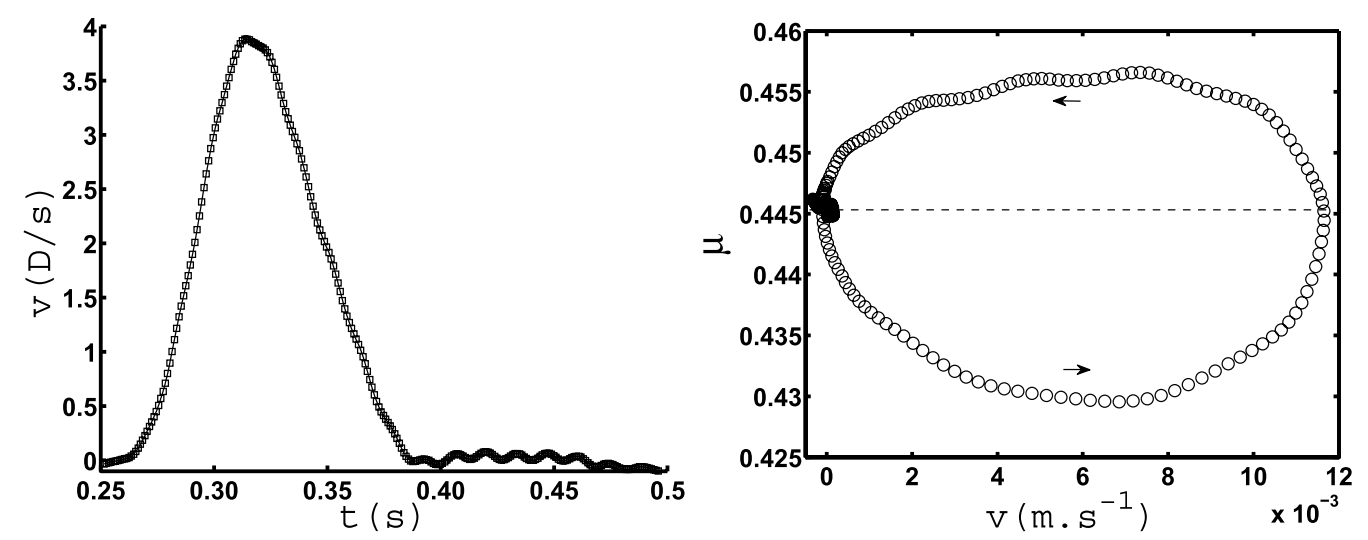

Figure 18. Left: evolution of the velocity, $v$, of type 1 grains during a precursor at $24^{\circ}$. Right: $\mu$ as a function of $v$.

The oscillations of the curve are due to $50 \mathrm{~Hz}$ fluctuations of the light emitted by the lamp used to track the grain positions. The acceleration and deceleration parts of the curve are seen to be quite different. The maximum velocity is about 4 diameter $\mathrm{s}^{-1}$. The friction force appears to be rather constant during the deceleration phase in contrast to the acceleration phase where the initial curvature indicates that the friction force decreases quickly when the grains begin to move. The evolution of the instantaneous friction coefficient, $\mu$, with the instantaneous velocity can be obtained from the derivative of the latter velocity. Assuming that a grain is submitted during its motion to its weight, $P=M g$, and to a friction force, $F_{\mathrm{f}}$, the instantaneous friction coefficient is indeed defined as

$$
\mu(t)=\frac{F_{\mathrm{f}}}{P_{N}}=\tan (\theta)-\frac{\dot{v}}{g \cos (\theta)}
$$

where $P_{N}=M g \cos (\theta)$ is the normal component of the weight and $\dot{v}$ is the instantaneous acceleration. The friction coefficient shows a hysteretic behaviour as a function of $v$ with a decrease during the acceleration step and an increase during the deceleration step. The decrease of $\mu$ when $v$ increases is consistent with previous results on stick-slip [28,29]. In fact, in these works sensitive measurements of the frictional forces produced by sheared layers of granular media were performed which showed that the instantaneous frictional force measured within individual slip events is a multivalued function of the instantaneous velocity. Nasuno et al $[28,29]$ observed further that localized microscopic rearrangements precede macroscopic slip events and that the accumulation of localized rearrangements leads to macroscopic creep. It is also useful to get information about the mechanism of propagation of an instability during a precursor event or during a localized rearrangement. To compare the mechanisms of cluster formation for these two cases, we plot in figure 19 the temporal evolution of grain velocities during a precursor (figure 19 right) and a localized rearrangement (figure 19 left) which occur at $24^{\circ}$ and $13^{\circ}$ respectively. Figure 19 shows the velocities of adjacent grains along the slope of the packing. The mechanism of propagation of localized rearrangements is a 'cascading mechanism' where destabilizations of grains at the surface cause rearrangements of their neighbours which are in turn destabilized. By contrast, the grains move in a synchronous manner during a precursor. 

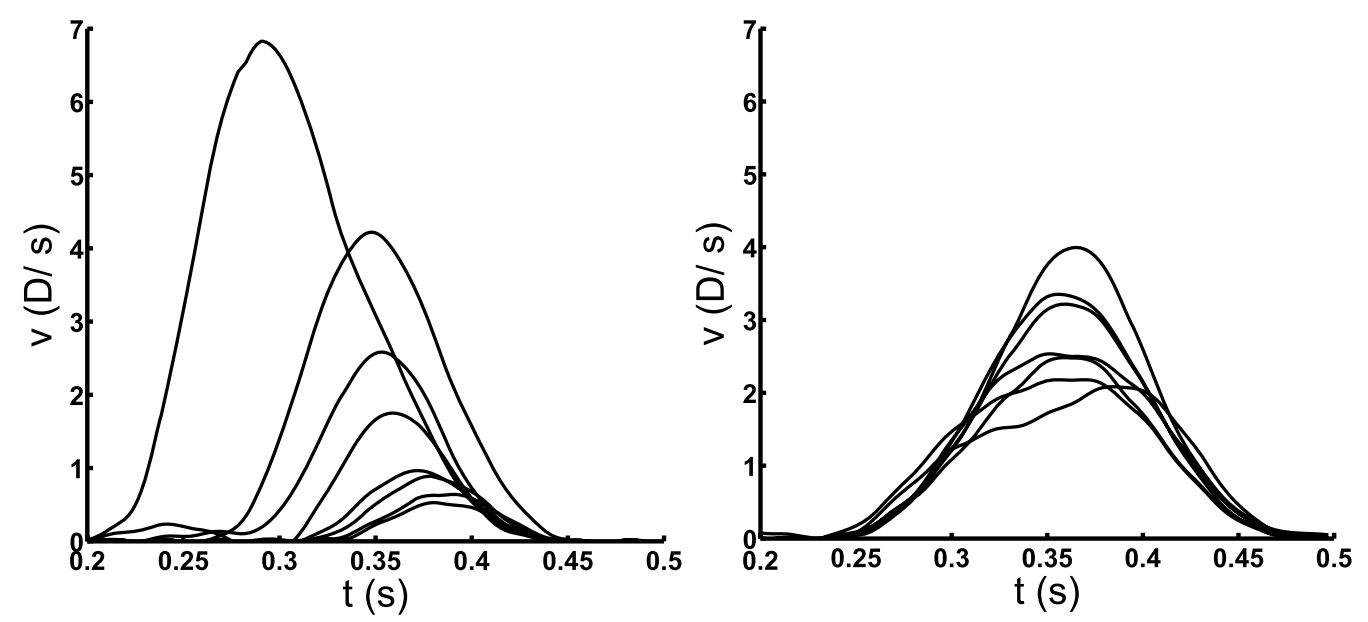

Figure 19. Evolution of the velocity, $v$, of adjacent grains of type 1 during a precursor at $24^{\circ}$ (right) and a localized rearrangement at $13^{\circ}$ (left).

This is in agreement with the hypothesis of a macroscopic instability of the whole surface and with the emergence of slidings (cracks) whose amplitudes are smaller than one grain diameter.

\section{Conclusions}

Experiments were performed on the unjamming transition of a slowly inclined 3D granular packing. The increase of the superficial activity of the packing with the inclination angle varies exponentially until the appearance of large events called precursors, a few degrees before the packing avalanches. This evolution is consistent with a simple trappingdetrapping model of grains. A numerical model based on the redistribution of local slopes shows that the exponential behaviour is robust. The precursors whose sizes are of the order of the area of the surface packing appear quasi-periodically and are strongly influenced by a confinement effect in the direction of the slope. This effect comes from the fact that they correspond to successive slidings of the surface of the packing similar to the stick-slip dynamics often observed in the frictional regime. In this context, we are led to assume that the precursors observed by Nerone et al [32], and described as large amounts of grains moving simultaneously at the surface, correspond to aftershocks of these large slidings. This conclusion explains why precursors are highly influenced by microscopic details of the interaction between grains. Indeed, the dynamics of these sliding events is controlled by the friction coefficient, the surface state, the ageing of contacts, etc.

It also appears that precursors are strongly influenced by the noise level. This noise seems to activate sliding at contacts between grains and make precursors vanish. These results complement those obtained experimentally for 3D packings by Kiesgen de Richter et al [20] and numerically by Staron et al [40,41], showing that the dynamics of an inclined granular packing ages gradually during repeated tilts associated with a hysteretic behaviour of the activity of rearrangements. These results allow a better identification of relevant parameters in other scientific fields such as soil stability or earthquake studies where this kind of non-stationary stick-slip behaviour appears. 


\section{Acknowledgments}

The study was supported in part by ANR grants STABINGRAM, ANR-05-BLAN-0273, NT-05-3-41489, and RFBR-PICS grant No 09-02-91071-CNRS. We thank A Faisant for technical help and D Bideau, Ph Boltenhagen, A Valance and H Mint Baba for helpful discussions.

\section{Appendix. Characterization of the critical angle: influence of the packing geometry}

We present below some experimental results of a study on the influence of the box length on the avalanche angle $\theta_{\mathrm{a}}$. Further, we discuss the relevance of this angle as an intrinsic parameter. The influence of the lateral confinement (box width) on granular flows [42] and avalanche angles [5] has been extensively studied. When the width is of the order of about ten grain diameters, the friction at walls modifies the momentum balance in all the packing and changes the value of the avalanche angle. To the best of our knowledge, we report here the first results on the influence of the longitudinal confinement of 3D granular packings on their critical angles.

\section{A.1. Avalanche angle as a function of the box length}

In this experiment, we used glass beads with the same diameter as those used before: $3 \mathrm{~mm}$. The length of the box was changed while keeping the height of the packing constant. Figure A.1 (upper part) shows the evolution of the critical angle $\theta_{\mathrm{a}}$ as a function of the length of the box L. Figure A.1 (lower part) presents the evolution of $\theta_{\mathrm{a}}$ as a function of $L / D$ where $D$ is the grain diameter. Data obtained by Mint Babah [25] in similar experimental conditions but with beads of $500 \mu \mathrm{m}$ are also shown. The critical angle decreases with $L$. The angle $\theta_{\mathrm{a}}$ is essentially independent of the box length for very large $L$ when $L / D \geq 1000$. By contrast, $\theta_{\text {a }}$ depends significantly on $L$ for small values of $L$. Moreover, the lower part of figure A.1 shows that $\theta_{\text {a }}$ depends on $D$ at $L / D$ fixed.

\section{A.2. Critical angle: an intrinsic parameter?}

A simple Coulomb criterion cannot explain the sensitivity of $\theta_{\mathrm{a}}$ to $L$. To explain our results we suppose the following.

- The fracture is localized at the surface of the packing; this means that a fracture appears at a height $h$ below the surface of the packing.

- The unstable upper part of the packing is submitted to three forces in the longitudinal direction at a given angle $\theta$ : the longitudinal component of the gravitational force $F_{\mathrm{p}}=\rho L h l \sin (\theta)$, the friction force $F_{\mathrm{f}}=-\mu_{\mathrm{s}} \rho g L l h \cos (\theta)$ and the reaction of the box $F_{\mathrm{r}}$, where $\mu_{\mathrm{s}}$ is an effective friction coefficient related to the macroscopic friction of a layer on another layer in the granular packing; $\rho$ and $l$ are the mean density and the width of the packing respectively.

- As a fracture criterion, we assume that the medium cracks when $F_{\mathrm{r}}$ exceeds a critical value $F_{\mathrm{s}}=\sigma_{\mathrm{s}} h l$ were $\sigma_{\mathrm{s}}$ is a critical stress. 

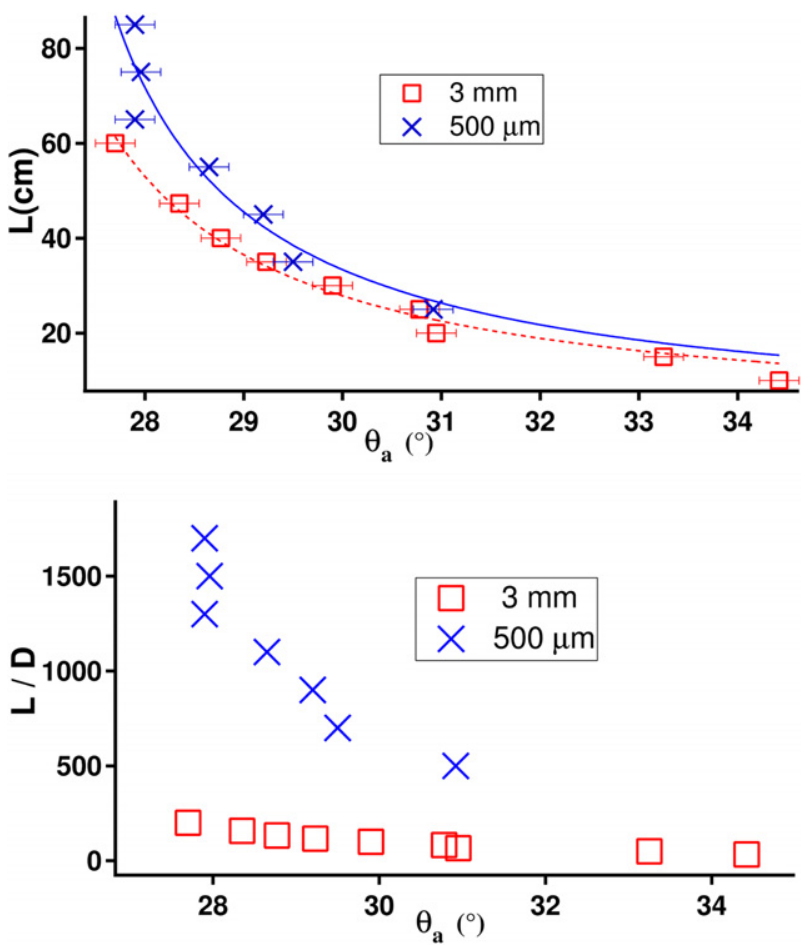

Figure A.1. Upper part: plot of $L$ as a function of $\theta_{\mathrm{a}}$ for bead diameters of $3 \mathrm{~mm}$ and $500 \mu \mathrm{m}$. The data have been fitted to equation (A.1). Lower part: $L / D$ as a function of $\theta_{\mathrm{a}}$. Each point is a mean over about ten independent experiments.

With this assumption, the medium cracks at the height $h$ when $\operatorname{\rho gh} L l\left(\sin \left(\theta_{\mathrm{a}}\right)-\right.$ $\left.\mu_{\mathrm{s}} \cos \left(\theta_{\mathrm{a}}\right)\right)=\sigma_{\mathrm{s}} h l$, then $L$ can be written as a function of $\theta_{\mathrm{a}}$ (equation (A.1)):

$$
L=\frac{\sigma_{\mathrm{s}} / \rho g}{\sin \left(\theta_{\mathrm{a}}\right)-\mu_{\mathrm{s}} \cos \left(\theta_{\mathrm{a}}\right)} .
$$

The curves of figure A.1 are well fitted to equation (A.1). For $3 \mathrm{~mm}$ beads, $\sigma_{\mathrm{s}} / \rho g=2.27 \mathrm{~cm} \pm 0.14$ and $\mu_{\mathrm{s}}=0.48 \pm 0.003$. For $500 \mu \mathrm{m}$ beads, $\sigma_{\mathrm{s}} / \rho g=2.42 \mathrm{~cm} \pm 0.48$ and $\mu_{\mathrm{s}}=0.49 \pm 0.008$. We find that values of $\sigma_{\mathrm{s}} / \rho g$ and $\mu_{\mathrm{s}}$ do not depend on the grain diameter in the range of diameters studied here.

This simple explanation shows that a local Coulomb criterion is not sufficient to explain the dependence of $\theta_{\mathrm{a}}$ on $L$. Here, we add a stricter macroscopic condition taking into account the fact that grains at the base of the packing feel the weight of the column of grains overhanging them in the length of the packing.

\section{References}

[1] Aegerter C M, Gunther R and Wijngaarden, Avalanche dynamics, surface roughening, and self-organized criticality: experiments on a three-dimensional pile of rice, 2003 Phys. Rev. E 67051306

[2] Aguirre M A, Nerone N, Calvo A, Ippolito I and Bideau D, Influence of the number of layers on the equilibrium of a granular packing, 2000 Phys. Rev. E 62738

[3] Aguirre M A, Nerone N, Ippolito I, Calvo A and Bideau D, Granular packing: influence of different parameters on its stability, 2001 Granular Matter 375

[4] Bak P and Tang C, Earthquakes as a self-organized critical phenomenon, 1989 J. Geophys. Res. 94635 
[5] Bolthenhagen Ph, Boundary effects on the maximal angle of stability of a granular packing, 1999 Eur. Phys. J. B 1275

[6] Bouchaud J P, Weak ergodicity breaking and aging in disordered systems, 1992 J. Phys. I 21705

[7] Boyer F, Guazelli E and Pouliquen O, Unifying suspension and granular rheology, 2011 Phys. Rev. Lett. 107188301

[8] Bretz M, Cunningham J B, Kurczynski P L and Nori F, Imaging of avalanches in granular materials, 1992 Phys. Rev. Lett. 692431

[9] Corwin E I, Jaeger H M and Nagel S R, Structural signature of jamming in granular media, 2005 Nature 4351075

[10] Deboeuf S, Dauchot O, Staron L, Mangeney A and Vilotte J-P, Memory of the unjamming transition during cyclic tiltings of a granular pile, 2005 Phys. Rev. E 72051305

[11] Debregas G, Tabuteau H and di Meglio J M, Deformation and flow of a two-dimensional foam under continuous shear, 2001 Phys. Rev. Lett. 87178305

[12] Fielding S M, Sollich P and Cates M E, Aging and rheology in soft materials, 2000 J. Rheol. 44323

[13] Fischer R, Gondret P and Rabaud M, Transition by intermittency in granular matter: from discontinuous avalanches to continuous flow, 1999 Phys. Rev. Lett. 103128002

[14] Gibiat V, Plaza E and De Guibert E, Acoustic emission before avalanches in granular media, 2008 J. Acoust. Soc. Am. 1233142

[15] Hamon D, Nicodemi M and Jensen H J, Continuously driven OFC: a simple model of solar flare statistics, 2002 Astron. Astrophys. 387326

[16] Hartley R R and Berhinger R P, Logarithmic rate dependence of force networks in sheared granular materials, 2003 Nature 421928

[17] Held G A, Solina D H, Keane D T, Haag W J, Horn P M and Grinstein G, Experimental study of critical mass fluctuations in an evolving sandpile, 1990 Phys. Rev. Lett. 651120

[18] Jaeger H M, Liu C-H and Nagel S R, Relaxation at the angle of repose, 1989 Phys. Rev. Lett. 6240

[19] Kabla A, Debregeas G, di Meglio J-M and Senden T J, X-ray observation of micro-failures in granular piles approaching an avalanche, 2005 Europhys. Lett. 71932

[20] Kiesgen de Richter S, Zaitsev V U, Richard P, Delannay R, Le Caer G and Tournat V, Experimental evidence of ageing and slow restoration of the weak-contact configuration in tilted $3 D$ granular packings, 2010 J. Stat. Mech. P11023

[21] Knight J B, Fandrich C G, Lau C N, Jaeger H M and Nagel S R, Density relaxation in a vibrated granular material, 1995 Phys. Rev. E 513957

[22] Liu A J and Nagel S R, Nonlinear dynamics: jamming is not just cool any more, 1998 Nature 39621

[23] Losert W, Bocquet L, Lubensky T C and Gollub J P, Particle dynamics in sheared granular matter, 2000 Phys. Rev. Lett. 851428

[24] Marty G and Dauchot O, Subdiffusion and cage effect in a sheared granular material, 2005 Phys. Rev. Lett. 94015701

[25] Mint Babah H, Etude expérimentale de quelques aspects de la problématique des dunes éoliennes: des processus d'avalanche à la statigraphie des dunes, 2010 PhD Thesis University of Rennes 1 www.sudoc.fr/146157036

[26] Monthus C and Bouchaud J P, Models of traps and glass phenomenology, 1996 J. Phys. A: Math. Gen. 293847

[27] Mueth D M, Debregeas G F, Karczmar G S, Eng P J, Nagel S R and Jaeger H M, Signatures of granular microstructure in dense shear flows, 2000 Nature 406385

[28] Nasuno S, Kudrolli A and Gollub J P, Friction in granular layers: hysteresis and precursors, 1997 Phys. Rev. Lett. 795

[29] Nasuno S, Kudrolli A, Bak A and Gollub J P, Time-resolved studies of stick-slip friction in sheared granular layers, 1998 Phys. Rev. E 582161

[30] Nerone N, Aguirre M A, Calvo A, Ipollito I and Bideau D, Surface fluctuations in a slowly driven granular system, 2000 Physica A 283218

[31] Nerone N and Gabbanelli S, Surface fluctuations and the inertia effect in sandpiles, 2001 Granular Matter 3117

[32] Nerone N, Aguirre M A, Calvo A, Bideau D and Ipollito I, Instabilities in slowly driven granular packing, 2003 Phys. Rev. E 67011302

[33] O'Hern C S, Langer S A, Liu A J and Nagel S R, Force distributions near jamming and glass transitions, 2001 Phys. Rev. Lett. 86111 
[34] Olami Z, Feder H J S and Christensen K, Self-organized criticality in a continuous, nonconservative cellular automaton modeling earthquakes, 1992 Phys. Rev. Lett. 681244

[35] Piegari E, Cataudella V, Di Maio R, Milano L and Nicodemi M, A cellular automaton for the factor of safety field in landslides modeling, 2006 Geo. Res. Lett. 33 L01403

[36] Pouliquen O, Scaling laws in granular flows down rough inclined planes, 1999 Phys. Fluids 11542

[37] Prado C P C and Olami Z, Inertia and break of self-organized criticality in sandpile cellular-automata models, 1992 Phys. Rev. A 45665

[38] Rosendahl J, Vekic M and Kelly J, Persistent self-organization of sandpiles, 1993 Phys. Rev. E 471401

[39] Sollich P, Rheological constitutive equation for a model of soft glassy materials, 1998 Phys. Rev. E 58738

[40] Staron L, Vilotte J-P and Radjai F, Preavalanche instabilities in a granular pile, 2002 Phys. Rev. Lett. 89204302

[41] Staron L, Radjai F and Vilotte J-P, Granular micro-structure and avalanche precursors, 2006 J. Stat. Mech. P07014

[42] Taberlet N, Richard P, Valance A, Losert W, Pasini J M, Jenkins J T and Delannay R, Superstable granular heap in a thin channel, 2003 Phys. Rev. Lett. 91264301

[43] Xu N V, Vitelli M, Wyart M, Liu A J and Nagel S R, Energy transport in jammed sphere packings, 2009 Phys. Rev. Lett. 102038001

[44] Yamamoto T, Yoschino H and Kawamura H, Simulation study of the inhomogeneous Olami-Feder-Christensen model of earthquakes, 2010 Eur. Phys. J. B 77559

[45] Zaitsev V, Richard P, Delannay R, Tournat V and Gusev V E, Pre-avalanche structural rearrangements in the bulk of granular medium: experimental evidence, 2008 Euro. Phys. Lett. 8364003

[46] Zhang Y and Campbell C S, The interface between fluid-like and solid-like behaviour in two-dimensional granular flows, 1992 J. Fluid Mech. 237541 